\title{
The sugarcane mitochondrial genome: assembly, phylogenetics and transcriptomics
}

\author{
Dyfed Lloyd Evans ${ }^{\text {Corresp., 1, } 2,3}$, Thandekile Thandiwe Hlongwane ${ }^{1}$, Shailesh V Joshi ${ }^{1,4}$, Diego M Riaño Pachón ${ }^{5}$ \\ 1 Plant Breeding, South African Sugarcane Research Institute, 170 Flanders Drive, Private Bag X02, Mount Edgecombe, Durban, KwaZulu Natal, South \\ Africa \\ 2 Cambridge Sequence Services (CSS), Waterbeach, Cambridge, CB25 9TL, United Kingdom \\ 3 Department of Computer Sciences, Université Cheikh Anta Diop de Dakar, BP 5005, Dakar, Sénégal \\ ${ }^{4}$ School of Life Sciences, College of Agriculture Engineering and Science, University of Kwa-Zulu Natal, Private Bag X54001, Durban, KwaZulu Natal, South \\ Africa \\ 5 Computational, Evolutionary and Systems Biology Laboratory, Center for Nuclear Energy in Agriculture, University of São Paolo, Piracicaba, São Paolo, \\ Brazil \\ Corresponding Author: Dyfed Lloyd Evans \\ Email address: dyfed.sa@gmail.com
}

Background. Chloroplast genomes provide insufficient phylogenetic information to distinguish between closely related sugarcane cultivars, due to the recent origin of many cultivars and the conserved sequence of the chloroplast. In comparison, the mitochondrial genome of plants is much larger and more plastic and could contain increased phylogenetic signals. We assembled a consensus reference mitochondrion with Illumina TruSeq synthetic long reads and Oxford Nanopore Technologies MinION long reads. Based on this assembly we also analyzed the mitochondrial transcriptomes of sugarcane and sorghum and improved the annotation of the sugarcane mitochondrion as compared with other species.

Methods. Mitochondrial genomes were assembled from genomic read pools using a bait and assemble methodology. The mitogenome was exhaustively annotated using BLAST and transcript datasets were mapped with HISAT2 prior to analysis with the Integrated Genome Viewer.

Results. The sugarcane mitochondrion is comprised of two independent chromosomes, for which there is no evidence of recombination. Based on the reference assembly from the sugarcane cultivar SP80-3280 the mitogenomes of four additional cultivars (R570, LCP85-384, RB72343 and SP70-1143) were assembled (with the SP70-1143 assembly utilizing both genomic and transcriptomic data). We demonstrate that the sugarcane plastome is completely transcribed and we assembled the chloroplast genome of SP80-3280 using transcriptomic data only. Phylogenomic analysis using mitogenomes allow closely related sugarcane cultivars to be distinguished and supports the discrimination between Saccharum officinarum and Saccharum cultum as modern sugarcane's female parent. From whole chloroplast comparisons, we demonstrate that modern sugarcane arose from a limited number of $S$. cultum female founders. Transcriptomic and spliceosomal analyses reveal that the two chromosomes of the sugarcane mitochondrion are combined at the transcript level and that splice sites occur more frequently within gene coding regions than without. We reveal one confirmed and one potential cytoplasmic male sterility factor in the sugarcane mitochondrion, both of which are transcribed

Conclusion. Transcript processing in the sugarcane mitochondrion is highly complex with diverse splice events, the majority of which span the two chromosomes. PolyA baited transcripts are consistent with the use of polyadenylation for transcript degradation. For the first time we annotate two cytoplasmic male sterility factors within the sugarcane mitochondrion and demonstrate that sugarcane possesses all the

PeerJ reviewing PDF | (2019:02:35349:1:1:NEW 5 Jul 2019) 
molecular machinery required for cytoplasmic male sterility and rescue. A mechanism of crosschromosomal splicing based on guide RNAs is proposed. We also demonstrate that mitogenomes can be used to perform phylogenomic studies on sugarcane cultivars. 


\section{The Sugarcane Mitochondrial Genome: Assembly,}

\section{Phylogenetics and Transcriptomics}

5 Dyfed Lloyd Evans ${ }^{1,2,3}$, Thandekile Thandiwe Hlongwane ${ }^{1}$, Shailesh Vinay Joshi ${ }^{1,4}$ and Diego M.

6 Riaño-Pachón ${ }^{5}$

$7{ }^{1}$ Plant Breeding, South African Sugarcane Research Institute, 170 Flanders Drive, Private Bag X02,

8 Mount Edgecombe, Durban, KwaZulu Natal, South Africa

$9 \quad{ }^{2}$ Department of Computer Science, Université Cheikh Anta Diop de Dakar, BP 5005, Dakar, Sénégal

$10{ }^{3}$ Cambridge Sequence Services (CSS), Waterbeach, Cambridge, CB25 9TL, UK

$11{ }^{4}$ School of Life Sciences, College of Agriculture, Engineering and Science, University of Kwa-Zulu

12 Natal, Private Bag X54001, Durban, KwaZulu Natal, South Africa

$13{ }^{5}$ Computational, Evolutionary and Systems Biology Laboratory, Center for Nuclear Energy in

14 Agriculture, University of São Paulo, Piracicaba, SP, Brazil

15

16 Corresponding Author:

17 Dyfed Lloyd Evans

18 Email address: dyfed.sa@gmail.com 


\section{Abstract}

20 Background. Chloroplast genomes provide insufficient phylogenetic information to distinguish between

21 closely related sugarcane cultivars, due to the recent origin of many cultivars and the conserved sequence

22 of the chloroplast. In comparison, the mitochondrial genome of plants is much larger and more plastic and

23 could contain increased phylogenetic signals. We assembled a consensus reference mitochondrion with

24 Illumina TruSeq synthetic long reads and ONT MinION long reads. Based on this assembly we also

25 analyzed the mitochondrial transcriptomes of sugarcane and sorghum and improved the annotation of the

26 sugarcane mitochondrion as compared with other species.

27 Methods. Mitochondrial genomes were assembled from genomic read pools using a bait and assemble methodology. The mitogenome was exhaustively annotated using BLAST and transcript datasets were mapped with HISAT2 prior to analysis with the Integrated Genome Viewer.

Results. The sugarcane mitochondrion is comprised of two independent chromosomes, for which there is no evidence of recombination. Based on the reference assembly from the sugarcane cultivar SP80-3280 the mitogenomes of four additional cultivars (R570, LCP85-384, RB72343 and SP70-1143) were assembled (with the SP70-1143 assembly utilizing both genomic and transcriptomic data). We demonstrate that the sugarcane plastome is completely transcribed and we assembled the chloroplast genome of SP80-3280 using transcriptomic data only. Phylogenomic analysis using mitogenomes allow closely related sugarcane cultivars to be distinguished and supports the discrimination between Saccharum officinarum and Saccharum cultum as modern sugarcane's female parent. From whole chloroplast comparisons, we demonstrate that modern sugarcane arose from a limited number of $S$. cultum female founders. Transcriptomic and spliceosomal analyses reveal that the two chromosomes of the sugarcane mitochondrion are combined at the transcript level and that splice sites occur more frequently within gene coding regions than without. We reveal one confirmed and one potential cytoplasmic male sterility factor in the sugarcane mitochondrion, both of which are transcribed 
43 Conclusion. Transcript processing in the sugarcane mitochondrion is highly complex with diverse splice

44 events, the majority of which span the two chromosomes. PolyA baited transcripts are consistent with the 45 use of polyadenylation for transcript degradation. For the first time we annotate two cytoplasmic male 46 sterility factors within the sugarcane mitochondrion and demonstrate that sugarcane possesses all the 47 molecular machinery required for cytoplasmic male sterility and rescue. A mechanism of cross-

48 chromosomal splicing based on guide RNAs is proposed. We also demonstrate that mitogenomes can be 49 used to perform phylogenomic studies on sugarcane cultivars.

52 Keywords: mitochondria; plastomes; sugarcane; phylogenetics; sugarcane origins; Saccharum cultum;

53 cytoplasmic male sterility, RNA splicing 


\section{Introduction}

55 Sugarcane ranks amongst the top-ten crop species worldwide. Sugarcane also provides between 60 and $70 \%$ of total world sugar output and is a major source of bioethanol (Reddy et al., 2008). Saccharum

57 officinarum L. is the type species for genus Saccharum L. Genus Saccharum, in the broad sense, (sensu 58 lato) consists of up to 36 species according to Kew's GrassBase (Clayton et al., 2006) or 22 validated species according to Tropicos (http://tropicos.org/Home.aspx). However, recent findings indicate that many of these species belong to different genera (Lloyd Evans, Joshi \& Wang, 2019) and that Saccharum sensu stricto (s.s.) [in the strict sense], consists of only four true species: Saccharum spontaneum L., Saccharum robustum Brandes \& Jeswiet ex Grassl, Saccharum officinarum and Saccharum cultum (Lloyd Evans \& Joshi, 2016). diversity in India (Sobhakumari, 2013). Before the 1780s, all sugarcanes arose from essentially sterile wild hybrids of Saccharum officinarum and Saccharum spontaneum (Artschwager \& Brandes, 1958; Irvine, 1999). During the 1800 s the new high-sucrose canes discovered in Polynesia supplanted these original hybrid canes. However, though productive and fertile, these cane varieties were susceptible to disease and from the 1920s, they were replaced by modern hybrid cultivars (complex hybrids of

71 Saccharum cultum Lloyd Evans and Joshi, Saccharum officinarum L. and Saccharum spontaneum L.

72 (Lloyd Evans \& Joshi, 2016)). As a result, the early history of the production of the first commercial sugarcane hybrids remains obscure, though hybrids generated in Java and Coimbatore, India,

74 predominate in the ancestry of almost all modern sugarcane hybrid cultivars. These new modern hybrids 75 possessed partly restored fertility, though pollen sterility varies amongst genotype and even in optimal conditions never reaches $100 \%$ (Subramanyam \& Andal, 1984). 
As most sugarcane cultivars were bred during the past 100 years, it has been hard to find a method

78 to reliably characterize the sugarcane breeding population phylogenetically. Though initially promising,

79 chloroplast genomes tend to be highly stable and there are insufficient sequence differences between

80 them to resolve the divergence of close sister cultivars (D Lloyd Evans, unpublished data).

Plant mitochondrial genomes are significantly different from their animal counterparts (Gualberto et al., 2014). Indeed, land plant mitochondrial genomes can vary in size between $187 \mathrm{kbp}$ in Marchantia polymorpha L. (Ohyama et al., 1986) to $11.3 \mathrm{Mbp}$ in Silene conica L. (Sloan et al., 2012). However, the

84 mitochondrial genome of the green alga Chlamydomonas reinhardtii Dangeard at 15 800bp is the 85 smallest yet assembled (Lister et al., 2003). The plasticity of mitochondrial genomes, leading to genome 86 expansion, arises primarily from repeat sequence, intron expansion and incorporation of plastid and 87 nuclear DNA (Turnel, Otis \& Lemieux, 2003; Bullerwell \& Gray, 2004). Moreover, plant mitochondria employ distinct and complex RNA metabolic mechanisms that include: transcription; RNA editing; splicing of group I and group II introns; maturation of transcript end and RNA degradation and translation (Hammani \& Giege, 2014). recombination events and dynamic genome rearrangements within a species leading to the generation of

94 Allen et al., 2007; Guo et al., 2016). In such cases, the complete genome is referred to as the master circle, with the DNA circles derived from recombination referred to as minicircles (subgenomic circles). Though the current convention is to represent the mitochondrial genome as a single DNA circle (often

97 resulting in duplication of repeat sequence in the final assembly), this is not always noted (Mower et al., 98 2012). 
101 recombination (e.g. Silene vulgaris (Moench) Garacke, S. noctiflora L., S. conica, Cucumis sativus L.)

102 (Sloan et al., 2012; Alverson et al., 2011) Functionally, plant mitochondrial genomes are unlikely to be 103 limited to a single origin of replication (Mackenzie \& McIntosh, 2006) (just as in their chloroplast 104 counterparts (Krishnan \& Rao, 2009)), though there has been only a single study analyzing in detail the 105 transcription of the plant mitochondrion in Petunia $\times$ hybrida hort, ex E. Vilm. (de Haas et al., 1991). The 106 mitogenome can be dynamic, with some plants possessing multipartite maps, typically containing fewer 107 than three chromosomes that can be assembled into circular, linear, branched or sigmoidal forms 108 (Gualberto \& Newton, 2017). In contrast, multichromosomal maps can contain tens of linear or circular 109 chromosomes (Sanchez-Puerta et al., 2017). Indeed, though the plant mitochondrial genome structure is often portrayed as a circle, micrograph 111 studies reveal that the true physical structure of the mitogenome appears to be a variety of circles, linear 112 molecules, and complex branching structures (Backert, Nielsen \& Börner1997; Backert \& Börner 2000).

113 While many plant species appear have a single master circle representation of their mitochondrial genome, 114 others are composed of more than a hundred circular chromosomes (Sloan et al., 2012) and two 115 independent chromosomes as in Allium cepa (Tsujimura et al., 2018). The precise mechanism of how 116 plant mitochondria replicate and maintain their DNA is not yet fully understood (Cupp \& Nielsen 2014) 117 but it is hypothesized that recombination-dependent replication plays a role, giving a functional role to the 118 repeat sequences often observed in mitochondria (Gualberto et al., 2014). However, studies in 119 Pyscomitrella patens (Odahara et al., 2015) revealed that two genes (RECA1 and the homolog of 120 bacterial RecG helicase, RECG) maintain mitochondrial genome stability by suppressing gross 121 rearrangements induced by aberrant recombination between short dispersed repeats. This is one major 122 reason why homologous recombination within the plant mitochondrial genome appears to be confined to 123 repeats greater than 1,000 bp in size (Arrieta-Montiel \& Mackenzie 2011). 
126 (Christensen, 2013). These long repeats, along with DNA shuffling between the nuclear and plastid

127 genomes can confound efforts to assemble plant mitochondrial genomes by introducing branch points

128 within the assembly graph that lead to multiple sequences including mitochondrial, nuclear and

129 chloroplast sequence being incorporated in an assembly. These effects, along with the relatively large

130 size of plant mitochondrial genomes, make them difficult to assemble. However, these effects in vivo

131 potentially introduce variable sequences that could be useful in comparing closely related cultivars.

132 Compared with the chloroplast and nuclear genomes, the mitochondrion is also unusual in that it

133 retains more bacterial-like transcript processing, whereby, in general, transcripts targeted for degradation

134 have poly-A extensions (Gagliardi et al., 2004). Though there may also be a secondary poly-A

135 mechanism protecting stress-induced transcripts (Adamo et al., 2008).

The plant mitochondrion is also typically responsible for a phenomenon known as Cytoplasmic

137 Male Sterility (CMS), a maternally inherited trait that typically results in a failure to produce functional

138 pollen or functional male reproductive organs (Suzuki et al., 2013). The phenomenon of CMS has been

139 reported in over 150 species of flowering plants (Carlsson et al., 2008). The highly recombinogenic,

140 repetitive nature of plant mitogenomes has been linked to CMS and, indeed, CMS is typically conferred

141 via chimeric genes whose generation has been associated with the presence of large repeats (Galtier,

142 2011). Typically CMS is counteracted by the presence of restorer-of-fertility $(R f)$ genes in the nuclear

143 genome (Huang et al., 2015). Functionally, there are three main routes to CMS in plants: mtDNA

144 recombination and cytonuclear interaction; regulation of CMS transcripts via RNA editing and direct

145 protein interactions whereby CMS protein transmembrane domains directly disrupt or alter the

146 permeability of the mitochondrial outer membrane, thus interfering with energy production (Chen et al., 147 2017). 
150 assembly of the mitochondrial genomes from the sugarcane cultivars LCP85-384, R570 and RB72343 as

151 well as Saccharum officinarum IJ76-514 from New Guinea. Extended annotation of the sugarcane

152 mitochondrial genome revealed a potential cytoplasmic male sterility factor that was a cognate of

153 ORF113 previously described in rice (Igarashi et al., 2013).

Transcript reads were mapped to the SP80-3280 mitochondrial chromosomes, revealing the spliceosome of sugarcane mitochondria. Poly-A baited transcripts were mapped to the Sorghum bicolor L. cv BTx623 mitochondrion, revealing mitogenomic regions tagged for degradation.

For phylogenetic analyses, mitochondrially-baited Illumina reads from Saccharum spontaneum SES234B and Miscanthus sinensis cv Andante were partially assembled against the sugarcane SP803280 template. The mitochondrial assembly from Sorghum bicolor BTx623 was employed as an outgroup.

162 the sugarcane mitochondrion is transcribed in its entirety and contains one confirmed and one potential

163 cytoplasmic male sterility factor as well as a functional copy of the chloroplast rbcL (rubisco large

164 subunit) gene. The sugarcane mitochondrion exists as two separate chromosomes without a master circle 165 and we present a guide RNA mechanism whereby transcripts can be trans-spliced between chromosomes. 166

\section{Materials and Methods}

\section{Sugarcane Mitochondrial Assembly}

NCBI was mined for assembled mitochondrial genomes and partial mitochondrial sequences from

170 the genera: Zea, Sorghum, Miscanthus and Saccharum. These sequences were used to bait reads from the

171 Saccharum hybrid SP80-3280 Illumina's TruSeq synthetic long read dataset (Table 1) using Mirabait 4.9 
172 (Chevreux, Wetter \& Suhai, 1999) with a k-mer of 32 and $n=50$. Baited reads were initially assembled

173 with Cap3, using parameters: -o 1000 -e 200 -p 75 -k 0 (Huang \& Madan, 1999). Assembled and

174 unassembled reads were blasted against the initial mitochondrial dataset with an e-value cut-off of $1 \mathrm{e}^{-9}$

175 (Camacho et al., 2008). All matching assemblies and reads were added to the read pool and a second

176 round of Mirabait read baiting was performed.

All baited reads were assembled with SPAdes (3.10) (Bankevitch et al., 2012) using default

178 parameters, but with all error correction options enabled. SPAdes contigs were blasted against the

179 mitochondrial dataset and all reads with matches were extracted. These were then blasted against a local

180 collection of Saccharum chloroplasts. All assemblies that had almost complete chloroplast coverage

181 were excluded. The final sugarcane mitochondrial assembly pool was baited against the Illumina TruSeq

182 synthetic long read pool using Mirabait again before running a second round of assembly with SPAdes.

183 The process above was repeated twice more.

184 At this stage, the longest contigs were tested for circularity with Circulator (Hunt et al., 2015).

185 This revealed a complete circular genome of $144639 \mathrm{bp}$. This sequence was labelled as 'potentially

186 complete' and was excluded from further assembly. The remaining contigs were run through four more

187 rounds of baiting and assembly. After these assembly rounds had completed circularity testing with

188 Circulator revealed a second complete chromosome of $300960 \mathrm{bp}$.

189

Using the two assembled mitochondrial chromosomes of SP80-3280, the mitochondrial genomes

190 of hybrid cultivars LCP85-384, RB72454 (Table 1) and S. officinarum IJ76-514 (Table 1) were

191 assembled using a methodology previously developed for chloroplast assembly (Lloyd Evans \& Joshi, 2016). Briefly, reads were extracted from the Illumina read pool using Mirabait with a baiting k-mer of

193 27. These reads were assembled using SPAdes with the SP80-3280 mitochondrion employed as an

194 untrusted reference (essentially to resolve repeats). Contigs were scaffolded on the corresponding SP80-

1953280 mitochondrial assembly and a second round of baiting and assembly was run, this time with a

PeerJ reviewing PDF | (2019:02:35349:1:1:NEW 5 Jul 2019) 
196 Mirabait k-mer of 31. After a second round of assembly, there were only a small number of short gaps

197 within the assembly. Excising a 2kbp region around the gap and using this for baiting and assembly

198 allowed this completed sequence to fill the gaps. Employing this approach, the two chromosomes of

199 LCP85-384 and RB72454 were assembled in their entirety. Chromosomes 1 and 2 of IJ76-514 were

200 partially assembled (both chromosomes contained gaps that could not be closed).

Though SRA datasets for Saccharum hybrid SP70-1143 existed in GenBank (Table 1), initial

202 assembly using the methods above failed to yield complete mitochondrial chromosomes. To improve coverage, five RNA-seq datasets were downloaded (Table 1). These are all single-end files and were used as an additional single-end dataset (with the --s option) of SPAdes. The combined dataset resulted in a complete hybrid assembly of both SP70-1143 mitochondrial chromosomes.

Subsequent to assembly, all assembled mitochondria were finished and polished with a novel pipeline. Raw reads from the SRA pool were mapped back to the assembly with BWA (Li \& Durbin, 2009), tagging duplicate sequences with Picard tools (http://broadinstitute.github.io/picard), optimizing the read alignment with GATK (McKenna et al., 2010) and finally polishing and finishing with Pilon 1.2.0 (Walker et al., 2014).

\section{1}

\section{Assembly of Sugarcane Cultivar R570 Mitochondrial Genome from PacBio}

\section{Sequel Reads}

214 cultivar genome data from JGI's Community Sequencing Program (Table 1) allowed for a novel

215 mitochondrial assembly based on long reads. As high read depth can be problematic for assembly, the

216 first 10 SRA files only were chosen for initial assembly. Reads were mapped to the assembled SP80-3280

217 sugarcane cultivar mitogenomes with minimap2 ( $\mathrm{Li}, 2018)$. Matched reads were converted from bam

218 format to FASTQ with SAMtools (Li et al. 2009). Reads were assembled with Canu and the final

219 assemblies were polished with Illumina reads (Table 1) using Apollo (Firtina et al. 2019).

PeerJ reviewing PDF | (2019:02:35349:1:1:NEW 5 Jul 2019) 


\section{Assembly Graph Optimization}

Assembly graphs can be key in determining how sequences are merged (or demonstrating that

222 there is no support for merging sequences). To obtain a high quality reference (and resolved) graph for the

223 sugarcane cultivar SP80-3280 the application Unicycler (Wick et al. 2017) was used to assemble its

224 mitochondrial genome. Unicycler helps to resolve repeats and loops in assembly graphs. A combination

225 of synthetic long reads (as long reads) and underlying Illumina short reads (as short reads) were employed

226 for assembly. Final assembly graphs were drawn and further resolved with Bandage (Wick et al. 2015).

227 The previously assembled SP80-3280 sugarcane mitochondrial genomes were mapped to the final graph

228 to help resolve the final collapsed repeats (5) so that a single path through each mitognome could be

229 drawn on the graph.

\section{Partial Assembly of Related Mitochondria}

231 Phylogenetic analyses require meaningful outgroups. For sugarcane this mans Saccharum

232 officinarum, Saccharum spontaneum and Miscanthus (Lloyd Evans et al. 2019). The mitochondrial

233 genomes of Saccharum spontaneum SES234B (Table 1) and Miscanthus sinensis cv Andante (gifted by

234 CCS, Cambridge, UK) were assembled using the sugarcane SP80-3280 mitochondrial chromosomes and

235 the Sorghum bicolor BTx623 (GenBank: NC_008360.1) mitochondrion as templates. Assembled contigs

236 were run through four rounds of baiting with Mirabait $(\mathrm{k}=31)$ and assembly with SPAdes. At the same

237 time, reads were mapped to the sugarcane mitochondrial genomes and the Sorghum mitochondrial

238 assembly with BWA (Li \& Durbin, 2009). Assemblies and mappings from Saccharum spontaneum

239 SES234B and Miscanthus sinensis cv Andante, along with the Sorghum mitochondrial assembly were

240 mapped to the sugarcane mitochondrial chromosomes using BLAST. These mappings were employed

241 for all subsequent phylogenetic analyses.

PeerJ reviewing PDF | (2019:02:35349:1:1:NEW 5 Jul 2019) 


\section{SP80-3280 Mitochondrial DNA Isolation and ONT MinION Sequencing and}

\section{Assembly}

244 Twenty just emerging SP80-3280 bud shoots were supplied on dry ice. The frozen tissue was

245 ground in a chilled mortar cold $\left(4^{\circ} \mathrm{C}\right)$ with grinding medium $(350 \mathrm{mM}$ mannitol, $30 \mathrm{mM}$ MOPS, $1 \mathrm{mM}$

246 EDTA, $50 \mu \mathrm{M}$ PVPP, $11.2 \mu \mathrm{M}$ L-cysteine; $\mathrm{pH}$ 7.6) at a volume of $2 \mathrm{ml}$ medium per gram of leaf tissue

247 (Strehle, Purfeerst \& Christensen, 2018). The resulting pulp was filtered through a double layer of

248 cheesecloth and the mortar was rinsed with cold grinding medium to recover residual leaf matter, which

249 was also passed through the cheesecloth. The crude product was filtered through a $0.45 \mu \mathrm{m}$ syringe filter

250 into clean microcentrifuge tubes and frozen at $-80^{\circ} \mathrm{C}$ for one hour. The mixture was thawed, brought to

251 room temperature and centrifuged in the cold at 5050g for two minutes. The supernatant was transferred

252 into clean tubes.

Enrichment and blocking probes (Table 2) were designed according to Shepard \& Rae (1997)

254 except that the biotinylated probe was 30nt long and blocking probes were 5 nt upstream and downstream

255 of the selection probe (synthesized by Thermo Fisher Scientific, Altrincham, UK). Probes were designed

256 against two conserved genes in the two mitochondrial chromosomes of sugarcane as well as two regions

257 that might represent fusion points between the two mitochondrial genomes - the latter to enrich for

258 master circles, if thy exist. An additional probe was designed against the $4 \mathrm{kbp}$ repeat in mitochondrial

259 chloroplast 1 to identify sub-genomic circles. Samples were divided into four aliquots and probes for mt1,

$260 \mathrm{mt} 2+$ master circle and $\mathrm{mt} 1$ subgenomic circles, respectively, were bound using the method of Shepard \&

261 Rae (1997). At the end of the hybridization reactions, $20 \mu 1$ washed streptavidin coated magnetic beads

262 (Thermo Fisher Scientific, Altrincham, UK) were added to each aliquot prior to shaking for 30 minutes

263 prior to magnetic bead isolation and washing. The supernatant was used for a second round of bead

264 microcapture. 
266 by Microcon spin columns (Sigma-Aldrich, UK) purification. The three main samples were combined

267 (but the mt1 sub-genomic circle baited DNA was kept separate) and the two resultant samples were used

268 directly for MinION sample preparation using the SQK-LSK109 Ligation Sequencing Kit with DNA

269 fragmenting. DNA was sequenced (9.4.1 chemistry) using MinKnow 2.0 a stacked analysis of two 10-

270 hour runs with a 10-minute wash was performed. Chiron (Teng et al. 2018), trained on a local sugarcane

271 database and run on an NVidia Tesla K80 24GiB within a Dell Power Edge server was employed for 272 basecalling.

'Fail' reads and reads $<500 \mathrm{kbp}$ in length were excluded. All remaining reads were taken forward

274 for assembly. The total read pool was assembled with Canu (Koren et al., 2017).

\section{Mitochondrial Genome Annotation}

276 Open Reading Frames (ORFs) were initially predicted using Open Reading Frame Finder

277 (https://www.ncbi.nlm.nih.gov/gorf/gorf.html). All tRNA genes were identified using tRNAscan-SE

278 (Schattner et al., 2005) In addition; genes and exons were extracted from the existing Sorghum and Zea

279 mays L. mitochondrial entries in GenBank. These features were mapped to the SP80-3280 assemblies

280 using Exonerate 2.2.0 (Slater \& Briney, 2005). A custom BioPerl script extracted the Exonerate mapped

281 features and compared them with predicted ORFs to determine confirmed genes. These genes were

282 further checked with the plant mitochondrial genome annotation program Mitofy (Alverson et al., 2010).

283 Repeats were identified using REPuter v3.0 (Kurtz et al., 2012) along with self-blasting the

284 mitochondrial chromosomes to themselves and each other. For chloroplast genes and other features, all

285 genes and features were extracted from the chloroplast genome of sugarcane cultivar RB72454 (NCBI:

286 LN849914) as well as the mitogenomes of Oryza rufipogon Griff. strain RT98C (NCBI: BAN67491)

287 (Igarashi et al., 2013) and the Oryza sativa L. Indica cv Hassawi mitochondrion (NCBI: JN861111)

288 (Zhang et al., 2012). Features were mapped with BLAST and manually added to the SP80-3280 
289 mitochondrial annotation files. The high quality annotation of the SP80-3280 mitochondrial genomes

290 was used as the basis for mapping features to the LCP85-384, RB72454, SP70-1143 and R570

291 assemblies using the Rapid Annotation Transfer Tool (RATT) (Otto et al., 2011). Completed and

292 annotated mitochondrial assemblies were deposited in ENA under the project identifier PRJEB26367.

293 The partial assembly of the IJ76-514 and the hybrid assembly of the SP70-1143 and the PacBio

294 assembly of R570 mitogenome were deposited in the Dryad digital repository (< doi available upon

295 acceptance $>$ ).

\section{CMS2 Identification, Sequencing and Analysis}

297

298

299

300

301

302

303

304

305

306

307

308

309

310

311

Identification of a novel transcript spanning potential chromosomal merge point $\mathrm{p} 4$ on chromosome 1 of the mitochondrial genome led to further analysis. BLAST using the DNA sequence and the potential protein sequence at NCBI yielded matches only against the Sorghum bicolor BTx623 and Tripsacum dactyloides cv Pete mitochondrial genomes. Domain analysis with InterProScan (Mitchell et al., 2019) demonstrated that the protein was formed from three key domains, none of which was completely represented in a single mitochondrial genome. The sequences from Saccharum hybrid SP803280, Tripsacum dactyloides cv Pete and Sorghum bicolor BTx623 were excised and used as baits for assembly in Chrysopogon zizanoides, Coix lacryma-jobi, Andropogon virginicus, Sarga versicolor, Miscanthus sinensis cv Andante and Saccharum spontaneum SES234B using SRA datasets detailed in Table 1.

To confirm the identity of five of the assembled sequences, primers were designed against the SRA assemblies and the corresponding mitochondrial regions were amplified and sequenced with ONT MinION (Supplementary Document 1). Expression of the potential CMS factors were confirmed by transcriptome mapping in Chrysopogon zizanoides, Tripsacum dactyloides, Sorghum bicolor and Saccharum hybrid cv SP80-3280.

Peer) reviewing PDF | (2019:02:35349:1:1:NEW 5 Jul 2019) 


\section{Sugarcane Chloroplast Genome Assembly}

313 The chloroplast of Saccharum hybrid cultivar SP70-1143 was assembled from NCBI sequence

314 read archive datasets as well as transcriptomic datasets (Table 1), as described previously (Lloyd Evans

315 \& Joshi, 2016). The R570 chloroplast was assembled from the following community SRA dataset:

316 SRR7517604. In contrast, the SP80-3280 chloroplast was assembled from TruSeq synthetic long reads

317 (Table 1), using our standard assembly pipeline, except for the following changes in Mirabait

318 parameters: $-\mathrm{k} 32-\mathrm{n} 150$. The SP80-3280 chloroplast was also assembled from transcriptomic data

319 (SRA: SRR1979660 and SRR1979664) (Mattiello et al., 2015). Transcriptomic assembly resulted in six

320 contigs covering all the chloroplast apart from the ribosomal RNA region, where there were 26

321 overlapping contigs. GC content (Supplemental Table S1) was used to identify contigs derived from the

322 chloroplast $(\mathrm{GC}$ content $=38.4 \%)$, which were made contiguous with CAP3 prior to integration into the

323 main assembly. Assemblies were finished and polished as described for mitochondrial assemblies. The

324 SP70-1143 short read assembly and SP80-3280 TruSeq synthetic long read assemblies were deposited in

325 the ENA under the project identifier PRJEB26685. The EMBL flatfiles corresponding to the genomic

326 assembly of R570, the transcriptomic assembly of SP80-3280 and the transcriptomic assembly of SP70-

3271143 can be obtained from Dryad ( $<$ doi available upon acceptance $>$ ).

328 Potential $\boldsymbol{R} \boldsymbol{f}$ Transcript and Mitochondrial Genome Stabilizer Transcript

329 Assembly and Sequencing

330 Restorer of Function (Rf) transcripts were identified from the Oryza literature (Gaborieau, Brown \&

331 Mireau, 2016; Fujii et al., 2014; Itabashi et al., 2011). The mitochondrial genome stabilizers RECA1 and

332 RECG helicases (Odahara et al. 2015) were identified from the Physcomitrella patens genome.

333 Orthologues of these genes were identified using the Ensembl Orthology (compara) interface (Viella et

334 al., 2009) or by Phytozome (Goodstein et al., 2011) BLAST analysis against the Miscanthus sinensis

335 genome assembly (Miscanthus sinensis v7.1 DOE-JGI, http://phytozome.jgi.doe.gov/). Transcripts and

PeerJ reviewing PDF | (2019:02:35349:1:1:NEW 5 Jul 2019) 
336 genes were assembled using a bait and assemble strategy (Lloyd Evans \& Joshi, 2017) against the SP80-

3373280 short read transcriptomic and TruSeq Synthetic Long Read genomic datasets (Table1). Primers

338 were designed (Table 3) to amplify as much of the transcript sequence as possible (as such the primers

339 were necessarily sub-optimal and could amplify multiple targets). Amplicons were concatenated with

340 rare cutter (eightmer) ligation adapters appended to the amplification primers and sequenced with Oxford

341 Nanopore Technologies MinION prior to assembly with CANU (Koren et al., 2017), as described

342 previously (see the SP80-3280 Mitochondrial DNA Isolation and ONT MinION Sequencing and

343 Assembly section, above). Sequences for three $R f$ transcripts were determined for the sugarcane cultivars

344 N22 and SP80-3280 and the two REC helicases were determined for SP80-3280. These sequences have

345 been deposited in ENA under the project identifier PRJEB26689.

\section{Transcriptomic Data Mapping}

347 Transcriptomic short read datasets (from the high depth SP80-3280 dataset SRA project:

348 PRJNA244522 (15 datasets) (Mattiello et al., 2015), a pooled cultivar dataset SRA: SRR849062 (though

349 containing SP80-3280 reads), a pooled tissue dataset SRA: SRR1974519 and a leaf dataset SRA:

350 SRR400035) were mapped to the SP80-3280 sugarcane mitochondrial chromosome assemblies and the

351 new SP80-3280 chloroplast assembly using BWA for unprocessed transcripts and HISAT2 (2.1.0) for

352 spliced transcripts (Kim, Langmead \& Salzberg, 2015). All mappings in SAM format were merged with

353 SAMtools (Li et al., 2009) prior to conversion to BAM and duplicate sequence removal with PICARD

354 and SAMtools prior to import into IGV (Integrative Genomics Viewer) (Thorvaldsdóttir et al., 2013).

355 The consensus sequence was exported from IGV, which was also employed to check for non-canonical

356 start codons and RNA-editing. Transcript counts at each base for the SP80-3280 data were exported with

357 the SAMtools 'depth' command prior to conversion to $\log _{10}$ and drawing on the mitochondrial genome

358 with Abscissa (Brühl, 2015).

PeerJ reviewing PDF | (2019:02:35349:1:1:NEW 5 Jul 2019) 
360 mapped to the SP80-3280 mitochondrial chromosomes and Sorghum bicolor BTx623 polyA baited

361 transcriptomic reads (SRA: ERR2097035; ERR2097063; ERR2097067; ERR3063529 and ERR3087932)

362 were mapped to the Sorghum bicolor mitochondrion (GenBank: NC_008360.1) initially with BWA. In all

363 cases paired end reads were used and reads where the mate did not map correctly or within the correct

364 distance were excluded from further analyses as these could represent genomic contamination. From the

365 total mapped read pool, reads only mapping to the forward strand were extracted with the SAMtools (Li

366 et al., 2009) command "samtools view -F $20<$ bam-file> > se-reads.sam".

367 Reads were converted back to fastq format and were re-mapped to the respective genomes with

368 HISAT2 (Kim, Langmead \& Salzberg, 2015), a fast read mapper that allows for long indels. Mapped

369 files were converted to BAM format with SAMtools and were imported into the IGV viewer

370 (Thorvaldsdóttir et al., 2013) for further analyses.

\section{Phylogenetic Analyses}

372 Assemblies of sugarcane mitochondrial chromosome 1 and chromosome 2 along with mappings

373 of S. officinarum chromosome 1 and partial chromosome 2 and Miscanthus, S. spontaneum and Sorghum

374 bicolor assemblies and contigs mapped to sugarcane mitochondrial chromosomes were aligned with

375 SATÉ 2.2.2 (Liu et al., 2009) using default options and the GTRGAMMA model, prior to manual

376 correction of the assembly. Missing sequence was represented by Ns. Regions of the assembly with over

$37720 \mathrm{nt}$ represented by a single sequence only were trimmed down to $10 \mathrm{bp}$ to reduce long branch issues.

378 Chromosome 1 alignments and Chromosome 2 alignments were merged with a custom Perl script.

379 Independent analyses were performed on the chromosome 1 dataset, chromosome 2 dataset and the

380 merged dataset. In all cases, the assemblies were partitioned into mitochondrial chromosomes and subset

381 into coding gene, tRNA + rRNA and non-coding partitions. Partition analyses with jModelTest2

382 (Darriba et al. 2012) revealed GTR $+\Gamma$ to be an acceptable model for all partitions.

PeerJ reviewing PDF | (2019:02:35349:1:1:NEW 5 Jul 2019) 
384 (Stamakis, 2006), using different seeds, were run with 100 replicates. Both runs yielded the same best 385 tree topology and this was used as the reference for all future analyses. Concatenated trees were 386 reconstructed using both maximum likelihood (ML) and Bayesian approaches and rooted on Sorghum 387 bicolor. The ML tree was estimated with RAxML using the GTR $+\Gamma$ model for all 5 partitions, and 6000 bootstrap replicates. The Bayesian tree was estimated using MrBayes v.3.2.1 (Ronquist \& Huelsenbeck 2003) using a gamma model with six discrete categories and partitions unlinked. Two independent runs with 25 million generations each (each with four chains, three heated and one cold) were sampled every 1,000 generations. Convergence of the separate runs was verified using AWTY (Nylander et al., 2008). The first six million generations were discarded as burn-in. The ML trees and the MB trees were mapped onto the best topology from the initial RAxML run with the SumTrees 4.0.0 script of the Dendropy 4.0.2 package (Sukumaran \& Holder, 2010).

Due to the large size of the combined $(\operatorname{chr} 1+\operatorname{chr} 2)$ and chromosome 1 datasets, divergence times on the smaller chromosome 2 alignment only were estimated using BEAST 2.4.4 (Drummond et al., 2012), on an 18-core server running Fedora 25, using four unlinked partitions (as above). However, as chromosome 1 and the combined partition gave the same tree topology, divergence times would not be expected to vary between datasets. The analysis was run for 50 million generations sampling every $1,000^{\text {th }}$ iteration under the GTR $+\Gamma$ model with six gamma categories. The tree prior used the birth401 death with incomplete sampling model (Drummond et al., 2012), with the starting tree being estimated 402 using unweighted pair group method with arithmetic mean (UPGMA). The site model followed an 403 uncorrelated lognormal relaxed clock (Drummond et al. 2006). The analysis was rooted to Sorghum 404 bicolor, with the divergence of Sorghum estimated as a normal distribution describing an age of $7.2 \pm 2$ 405 million years ago (Lloyd Evans \& Joshi, 2016). Convergence statistics were estimated using Tracer v.1.5 406 (Rambaut et al., 2013) after a burn-in of 15,000 sampled generations. Chain convergence was estimated 407 to have been met when the effective sample size was greater than 200 for all statistics. Ultimately, 
40830,000 trees were used in SumTrees to produce the support values on the most likely tree (as determined 409 above) and to determine the $95 \%$ highest posterior density (HPD) for each node. All final trees were 410 drawn using FigTree v.1.4.0 (http://tree.bio.ed.ac.uk/software/figtree/) prior to finishing in Adobe

411 Illustrator. Final alignments and phylogenetic trees are available from the Dryad digital repository $(<$ DOI 412 available on acceptance>).

\section{Mitochondrial and Chloroplast Comparisons}

414 Mitochondrial and chloroplast chromosome comparisons (within and between sugarcane cultivars) 415 were performed with NCBI BLAST (Altschul et al., 1990), Mauve (Darling et al., 2004) and EMBOSS

416 Stretcher (Rice, Longden \& Bleasby, 2000). EMBOS Stretcher output was analyzed with a custom Perl 417 script to detect and quantify substitutions, insertions and deletions between the two genomes.

\section{GC Content Analyses}

419 GC content varies between the chloroplast, mitochondrion and the nuclear genome. We used our 420 assemblies to compare GC content between related mitochondria, related chloroplasts, the assembled 421 genomes of Sorghum and maize and the synthetic long read pool of sugarcane (excluding mitochondrial 422 and plastome reads) using the EMBOSS cusp application. The data obtained from this study was used to 423 ensure that our mitochondrial assembly arose only from mitochondrial data and to examine introgression 424 of sequence from the chloroplast and nuclear genome into the mitochondrion of sugarcane. Results are 425 presented in Supplemental Table S1. 


\section{Target Peptide Analysis}

The full length transcripts for the genes RECG and RECAl were translated with ExPASy translate

428 (https://web.expasy.org/translate/) prior to submission to the following web-based target peptide analysis

429 tools: TargetP 1.1 (Emanuelsson et al., 2007); TPpred 2.0 (Savojardo et al., 2014); and LOCALIZER 1.0

430 (Sperschneider et al., 2017).

\section{Gene Expression Analysis}

To determine the expression of mitochondrial genes, the gene region was excised from the genome and SP80-3280 transcriptomic reads (Table 1) were mapped to the genome with BWA. Duplicate reads were marked with GATK and removed prior to indexing and visualization in IGV. Mapped reads were counted with SAMtools. nad6 was employed as a positive control reference and a 100bp non-coding region from the SP80-3280 sugarcane mitochondrial chromosome 1 was employed as a background reference. Mapped read counts were normalized against gene length and were expressed as fold counts against the background region. Analysis of rbcL was more complex, as rbcL was derived from the chloroplast. As such high depth chloroplastic reads could confound expression analysis. However, sequence comparisons indicate that the $\mathrm{C}$-terminal of rbcL is different in the chloroplast and mitochondrial copies, meaning that this region could be used for expression analyses.

\section{Transposable Element Analyses}

444 the Poaceae database as query for the Genetic Information Research Institute

445 (http://www.girinst.org/censor/index.php) Censor application (Kohany et al., 2006).

447 species sequences. Using BioPerl (Stajich et al. 2002) features were extracted and transposable elements

448 were identified by keyword search. This yielded 327 unique transposon sequences, which were subset 
449 into 170 unique potentially autonomous transposable elements. These were further classified into eight of

450 the eleven plant transposon orders/families prior to being mapped to the sugarcane mitochondrial genome

451 sequences using BLASTN for DNA transposons and psi-BLAST for translatable transposons (Altschul et

452 al. 1997). BLAST analysis returned no hits to the sugarcane mitogenomes (not even partial hits). As a

453 result, unique sequences were converted to profile hidden Markov models (HMMS) using HMMER

454 (Eddy 1998).

455

The PiRATE pipeline (Berthelier et al. 2018) was installed as a virtual machine within Galaxy

456 (Afgan et al. 2018). Comparisons of the profile HMMs with the profile HMMs within PiRATE revealed

457 only two partially divergent HMMS within the sugarcane dataset. These were added to the PiRATE

458 HMM collection. The PiRATE pipeline was run as specified by the authors and all hits were combined

459 with the initial hits from Censor.

\section{Molecular Modelling of rbcL}

461

The protein sequences of sugarcane chloroplast rbcL (rubisco large subunit) and mitochondrial

462 rbcL were submitted to the Phyre ${ }^{2}$ server (Kelley et al.; 2015) for homology modelling. PDB files from

463 Phyre $^{2}$ intensive modelling were downloaded and prepared for molecular dynamics (MD) simulation

464 using the Protein Preparation Wizard of the Maestro molecular modelling software (v.9.6; Schrödinger,

465 Inc.). The model included all hydrogen atoms from the start, but the polar interactions of the His residues

466 were manually checked and the protonation states selected to optimize the hydrogen bond network.

MD simulations were performed to confirm that the 3D structure was stable without unfolding or

468 any significant changes in secondary structure. The Groningen Machine for Chemical Simulations

469 (GROMACS) (Abraham et al. 2015) with the CHARMM force field was employed for this purpose and

470 solvated our model in a cubic box with TIP3P water. The system was charge equilibrated with 8 sodium

471 ions before being energy minimized. After energy minimization, the systems were equilibrated by

472 position restrained molecular dynamics at constant temperature of $300 \mathrm{~K}$ and a constant pressure of $1 \mathrm{~atm}$ 
473 for about 100 ps before running a 200 ns molecular dynamics simulation using the CHARMM force-field.

474 The final models were compared with each other and with the original spinach template to ensure

475 conformational stability.

476 Final models were imported into USCS Chimera (Pettersen et al., 2004) and were superimposed

477 with the MatchMaker tool and RMSD differences were determined from the Reply Log panel.

478 


\section{Results}

\section{Mitochondrial Genome Assembly and Annotation}

An iterative approach was used to assemble the mitochondrial genome of Saccharum hybrid cv.

482 SP80-3280 using Illumina's TruSeq synthetic long reads. This resulted in the assembly of two

483 mitochondrial chromosomes: one of 144639 bp and one of $300960 \mathrm{bp}$ (Figure 1). Average read depth on

484

485

486

487

488

489

490

491

492

493

494

495

496

497

498

499

500

501

502

both chromosomes was $12.4 \mathrm{x}$. No reads were found that directly linked the two chromosomes, indicating that the sugarcane mitochondrion exists as two separate chromosomes without a master circle. Whilst there were a large number of repeats within each chromosome, few repeats were found to be common between both chromosomes (Table 4). This makes it unlikely that the chromosomes can recombine to form a master circle.

In addition, the mitochondrial genomes of LCP85-384 and RB72454 were assembled from Illumina short read data (Table 1). We also attempted assembly of the mitogenome of cultivar SP70-1143. There was insufficient coverage from nuclear sequence to completely assemble the two mitochondrial chromosomes of this $S$. officinarum isolate. As a result, a hybrid approach was attempted, adding five RNA-seq datasets to improve overall coverage. This resulted in the complete assembly of the two SP701143 mitochondrial chromosomes. The mitochondrial genome of sugarcane cultivar R570 was assembled from PacBio Sequel long reads using Canu.

All mitochondrial genomes had a $15 \mathrm{kbp}$ direct repeat sequence and a $4 \mathrm{kbp}$ inverted repeat on chromosome 1 (Figure 1). Full annotation of the genomes (based on previous mitochondrial annotations, mapping chloroplast genes and mapping additional genes from rice and maize mitogenomes) revealed 72 unique open reading frames plus 26 duplicate copies, 14 complete chloroplast genes and 27 partial chloroplast gene fragments. Of these, 64 genes are encoded by a single exon and eight genes are encoded across multiple exons. Moreover, trans-splicing of group II introns was observed in three genes: nadl, nad2 and nad5. The genes nad2 and nad5 have exons split between chromosome 1 and chromosome 2 (a

Peer] reviewing PDF | (2019:02:35349:1:1:NEW 5 Jul 2019) 
503 similar phenomenon is seen in Silene vulgaris (Sloan et al., 2012)). Sugarcane mitochondrial genomes

504 had the same gene content as sorghum, with the exception of $\operatorname{trn} L-C A A$ and rbcL-cp, which are present in 505 sugarcane, but absent from sorghum.

506 Comparisons of the mitochondrial assembly of SP80-3280 with the chloroplast genome assembly 507 from the same cultivar revealed that seven of the total tRNA genes plus 14 other genes were derived from 508 the chloroplast genome, mostly present in large sections of transferred DNA.

\section{Assembly Graph Analysis}

511 read data yielded a single almost completely resolved assembly graph (Figure 2a). Mapping the previous

512 assembly of Khon Kaen 3 cultivar mitogenomes demonstrated that the two mitogenomes of SP80-3280

513 were assembled in their entirety and with the same organization. However, the assembly graph revealed

514 five points ( $\mathrm{p} 1$ to $\mathrm{p} 5$ in Figure $2 \mathrm{a}$ ) via which the two mitogenomes could possibly be combined into a

515 master circle. All assemblies using short read data yielded an identical assembly graph. Fortuitously,

516 during the course of this experiment, over 500Gbase of PacBio long read data for the sugarcane cultivar

517 R570 were released as part of JGI's community sequencing initiative. Reads were downloaded;

518 mitochondrial sequences were baited with minimap2 prior to assembly with Canu. The assembly graph

519 (Figure 2b) revealed two mitochondrial chromosomes with no reads linking them.

520 BLAST comparisons of the two mitochondrial genomes of SP80-3280 revealed 111 small regions

521 in common between chromosome 1 and chromosome 2 of the mitochondrial genome. 106 of these could

522 be excluded from joining the two chromosomes into a mitogenomes, as they are smaller than the Illumina 523 read lengths and no reads crossing these common sequences to join the two chromosomes together were 524 detected in any Illumina or long read datasets. Moreover, the small regions duplicated between sugarcane 
525 mitochondrial chromosomes are variable between sugarcane cultivars (Table 4) and are often imperfect

526 (i.e. the sequences are not identical).

527 To further confirm that the two mitochondrial genomes of sugarcane do not form a master circle,

528 the two mitochondrial genomes and the putative master circle were isolated using probes and microbead

529 extraction. ONT MinION sequencing and Canu assembly yielded two mitochondrial genomes (Fig 2c)

530 with no reads joining the two mitochromosomes.

531 In addition, to further exclude the presence of a master circle, sequences corresponding to the five 532 potential chromosomal merge points were extracted from the assembly graph. Minimap2 was employed 533 to extract all reads containing these sequences. Sequences corresponding to all potential paths through the 534 join points were extracted from the graph and the baited reads were mapped to these sequences. In no 535 case were any reads found that joined the two chromosomes into a master circle.

\section{GC Content Analyses}

Analyses of GC content across a range of Andropogoneae chloroplast assemblies (Supplemental

538 Table S1) reveal a very narrow GC range of 38.4-38.5\%. Mitochondrial GC analysis also reveals a

539 narrow range of GC values from 43.07-43.93\%. Though fewer genomes have been assembled, the

540 available data shows GC percentages ranging from 41.4-42.7\%. Thus, chloroplast, genomic and

541 mitochondrial data have unique GC signatures that can be employed to analyse cross-contamination

542 within the genomes.

\section{Mitochondrial Assembly of Ancestral and Outgroup Species}

Assembly of the Saccharum spontaneum SES234B mitochondrial genome was attempted. Large

545 contigs were obtained, demonstrating considerable sequence conservation with the sugarcane hybrid

546 assemblies. Examining the assembly graphs for the S. spontaneum cv SES234B mitogenome revealed that

547 there were multiple reads joining chromosome 1 and chromosome 2 as based on the sugarcane hybrid 
548 assemblies. This indicates that either the S. spontaneum mitochondrion exists as a single circular genome

549 or there is a different organization of this species' mitochondrial chromosomes. As a result, we were not

550 able to completely assemble the mitogenome of $S$. spontaneum. As a compromise, the assembled $S$.

551 spontaneum mitochondrial contigs were mapped to the sugarcane chromosome 1 and chromosome 2

552 assemblies. This mapping was subsequently used for phylogenetic analyses.

An attempt at assembling reads for Miscanthus sinensis cv Andante revealed a similar pattern to

554 that of $S$. spontaneum, again indicating that the mitochondrion of this species also exists as a single

555 chromosome. Again, M. sinensis contigs were mapped to the sugarcane chromosome 1 and chromosome

5562 assemblies for subsequent use in phylogenetic analyses. Assembly graphs for the S. spontaneum and

557 Miscanthus assemblies are available as Supplemental Document S1 and show that incomplete assembly

558 was du to complexities in the assembly graphs rather than lack of genome coverage (datasets have more

559 than 14 Gbase reads) (Table 1).

560

Assembly of S. officinarum cv IJ76-514 was attempted, using our previous chloroplast assembly

561 for this cultivar (Lloyd Evans \& Joshi, 2016) all reads mapping to the chloroplast were removed with

562 BWA and SAMtools. The remaining reads were baited and assembled based on the SP80-3280

563 mitochondrial genome assembly. It took five rounds of baiting and assembly to fully assemble

564 chromosome 1 (apart from six small gaps), but after 10 rounds chromosome 2 still had significant gaps.

565 This could mean low coverage of certain genomic regions, but it also indicates more sequence variation

566 than had previously been reported.

\section{Phylogenomic Analyses}

568 BLAST analysis of our assembled SP80-3280 mitochondrial chromosomes against the assembled

569 mitochondrial genome of Sorghum bicolor BTx623, revealed that $345 \mathrm{kbp}$ of its $468 \mathrm{kbp}$ genome is

570 represented in our assembly, although, substantially rearranged. Thus, considerable portions of the total

571 mitochondrial repeat sequences are shared between the two species. This includes $3 \mathrm{kbp}$ of the $4 \mathrm{kbp}$

PeerJ reviewing PDF | (2019:02:35349:1:1:NEW 5 Jul 2019) 
572 inverted repeat and the entire $15 \mathrm{kbp}$ direct repeat, though split into two parts in sorghum, with the entire

573 repeat existing as only a single copy in the Sorghum mitogenome. This indicates that our strategy of

574 mapping assembled contigs from Miscanthus and Saccharum spontaneum onto the sugarcane assembly

575 is valid and results in accurate sequence for phylogenetic analyses.

576 Mitochondrial chromosome assemblies of the sugarcane hybrids: SP80-3280, Khon Kaen 3,

577 LCP85-384, RB72343, R570 and SP70-1143 were separately aligned to the two chromosomes from $S$.

578 officinarum IJ76-514 as well as the mapping of the sorghum mitogenome to the two sugarcane

579 chloroplasts and the mappings of S. spontaneum and M. sinensis contigs to the two chromosomes of

580 sugarcane. Each chromosome was aligned independently, prior to both alignments being merged.

581 Maximum likelihood analyses of chromosome 1, chromosome 2 and the combined dataset revealed

582 exactly the same tree topology (Figure 3). The chromosome 1 and chromosome 2 alignments were taken

583 further for ML bootstrap and BI support determination. Both analyses revealed 100\% support for all

584 branches. The data for chromosome 2 only is shown in Figure 3, as only this dataset was employed for

585 BEAST analyses to generate a chronogram. The phylogeny shows the expected topology and is consistent

586 with our previous studies (Lloyd Evans \& Joshi, 2016; Lloyd Evans, Joshi \& Wang, 2019). We also

587 clearly see the expected ancestral relationships between SP70-1143, SP80-3280 and Khon Kaen 3.

\section{Transcriptomic Read Mapping}

589 High depth RNA-seq data were available for sugarcane cultivar SP80-3280 (Table 1) and were

590 mapped to the mitochondrial genome for spliceosome analysis. Unfortunately, there were insufficient

591 polyA-baited reads to allow mapping to the sugarcane mitochondrion. As a result mapping of polyA-

592 baited reads was performed to the Sorghum bicolor BTx623 mitochondrial genome instead.

593 After pre-processing to ensure both reads of paired end data mapped to the appropriate

594 mitochondrial genome, reads were converted to forward strand only. These reads were re-mapped with

595 HISAT2 and imported into IGV prior to analysis.

PeerJ reviewing PDF | (2019:02:35349:1:1:NEW 5 Jul 2019) 
597 pattern of splicing events, many spanning the two chromosomes (Figure 4). The most common splicing

598 event joined the start of chromosome 1 with the start of chromosome 2. Internally, splicing events were

599 from one locus hotspot to another locus hotspot that spanned a few hundred to a few thousand bases. Thus

600 splicing events were not targeted to a few bases as is typical in eukaryotic genomes. In addition, of 222

601 splicing events (only counting splice sites with $>=10$ reads mapped) 110 (49.55) were inside coding

602 sequences - which is almost half — an unexpectedly high number. The full analysis of splice sites in the

603 SP80-3280 mitochondrial chromosomes in given in Supplemental Table S2.

604

Compared with other plant genomes, mitochondria are unusual in that they retain much (though

605

606

607

608

609

610

611

612

613

614

615

616

617

618

619

620

not all) of their $\alpha$-proteobacterial antecedents' processing (Gagliardi et al., 2004). Indeed, under nonstressed conditions mitochondria add poly-A tails to those transcripts marked for degradation. To examine this process polyA-baited reads were mapped to the Sorghum bicolor BTx623 mitochondrial genome. As can be seen from Figure 5, polyA baited reads map to distinct 'islands' within the sorghum mitochondrial genome. Examining these islands, of the 35 identified, only five contained genes annotated in the Sorghum bicolor mitogenome. However, when the chloroplast and nuclear genomes were included in searches along with mitochondrial gene duplications an additional 24 genes were identified. The remaining polyA tailed regions were all repeat regions, intronic regions and intragenic regions. The full analysis of polyA read islands mapped to genes is provided in Supplemental Table S3.

A $1 \mathrm{kbp}$ region around the five potential chromosomal merge points were excised and mapped back to the genome to identify annotated genes and both long and short read transcriptomic data were mapped to these sequences. Both annotation and transcript mapping confirmed that $\mathrm{p} 1$ was overlapped by the mitochondrial gene rps 4 on chromosome 2, p4 was overlapped by $c c m F c$ on chromosome 1 and $\mathrm{p} 5$ was overlapped by nad6 on chromosome 2 . Transcript mapping revealed a novel sequence supported by 50 transcripts covering $\mathrm{p} 4$ on chromosome 1 . Translation of this sequence revealed it to have a single hit in NCBI BLAST, corresponding to a Sorghum bicolor hypothetical protein (NCBI: OQU77742.1) whilst

PeerJ reviewing PDF | (2019:02:35349:1:1:NEW 5 Jul 2019) 
621 nucleotide BLAST mapped to the Sorghum bicolor BTx623 mitochondrion, though the region is currently

622 unannotated.

\section{CMS Region Origins and Expression}

624

625

626

627

628

629

630

631 632 proteins.

633

634

635

636

637

638

639

640

641

642

643

Mapping of the Oryza rufipogon strain RT98C (NCBI: BAN67491) mitochondrial features to the sugarcane mitochondrion revealed unexpected homology between ORF113 in O. rufipogon and a putative 345nt Open Reading Frame (ORF) in the sugarcane mitogenome (chromosome 1) see Figure 1. ORF113 is labelled as a 'candidate cytoplasmic male sterility gene' as identified by Igrashi et al. (2013) and which has subsequently been demonstrated to be the causative agent of CMS in the RT98A (without restorer functionality) line of $O$. rufipogon (Toriyama et al., 2013). This novel gene was named CMS1, the expression of which is supported by transcriptomic analysis. Domain analysis (Figure 6a) revels that the protein is a fusion of a transmembrane domain with a C-terminal domain, which is a signature of CMS

Additional BLAST analyses revealed that a pseudogene corresponding to ORF113 was present in the maize mitochondrial genome but that an orthologue was not present in the Sorghum bicolor mitochondrion. The complete CMS sequence was identified in the S. officinarum IJ76-514 mitochondrial assembly as well as the mapped assembly of Miscanthus sinensis. However, it was not detected in a complete and translatable form in the mapped assembly of S. spontaneum despite the region that contains this sequence being present in the $S$. spontaneum mitogenome contigs.

The novel gene discovered in potential sugarcane mitochromosome crossover point p4 (Figure 2a) is a fusion protein; with all the characteristics of a CMS protein (Supplemental Document S2, Figure 6) which transcriptomic analysis demonstrates is expressed. Sequence assembly reveals that this gene is present in genera Sarga, Miscanthus and S. spontaneum cultivar SES234B as well as all the sugarcane cultivars analysed. This novel gene is not present in the mitogenomes of maize. Domain analysis and

Peer] reviewing PDF | (2019:02:35349:1:1:NEW 5 Jul 2019) 
644 alignment of all the assemblies with nad6 from the mitogenomes of Khon Kaen 3 revealed that the protein

645 was a fusion of half the nad6 gene with a non-cytoplasmic 5' domain and a long cytoplasmic 3' domain,

646 with the nad6 region donating transmembrane regions to the fusion protein (Supplemental Document S2).

647 Potential $\boldsymbol{R} \boldsymbol{f}$ Transcript and Mitochondrial Genome Stabilizer Transcript

648 Assembly and Sequencing

649 Homology analysis revealed three potential restorer of function $(R f)$ genes in sorghum and two

650 mitochondrial stabilizing genes. These were assembled in the SP80-3280 cultivar of sugarcane, from

651 which primers were designed. These were employed to amplify and sequence the transcripts

652 corresponding to these genes from sugarcane cultivars. Target peptide analysis demonstrated that both of

653 the genome stabilizer transcripts are directed to the mitochondrion.

\section{Transposable Element Analysis}

655 Censor (Kohany et al., 2006) analyses revealed 114 potential transposable element fragments in

656 chromosome 1 and 48 potential transposable elements in chromosome 2 . The coordinates of the

657 transposable elements in chromosome 1 and chromosome 2 of the SP80-3280 mitochondrial genome are

658 given in Supplemental Table S4. Extraction of transposable elements from Saccharum species sequences

659 deposited in NCBI and mapping to the sugarcane mitogenomes revealed no matches. Even converting

660 the sequences to profile HMMs only yielded two profiles slightly divergent from those in PiRATE,

661 though running the pipeline revealed no additional transposon fragments over and above those

662 discovered by Censor. Though there are many fragments of transposons within the mitochondrial

663 genome, none are functional and all are degraded from their original genomic ancestors.

\section{Chloroplast Assembly and Analyses}

665 The currently published SP80-3280 chloroplast was assembled in 2002 (Calsa Jr et al., 2004).

666 The state of the art in terms of chloroplast assembly and sequence finishing has moved on considerably 
667 during the intervening decade and a half. We re-assembled the SP80-3280 chloroplast from Illumina's

668 TruSeq synthetic long reads, using our novel sequence-finishing pipeline for assembly polishing.

669 Analyses showed that our assembly differed from the GenBank accession by only 8 substitutions and a

670 single insertion (Table 2). This is compatible with the number of errors predicted by Hoang et al. (2015).

671 To see if this was typical or unusual, we also assembled the SP80-3280 chloroplast from transcriptomic

672 data, as well as assembling the chloroplast of the closely related cultivar SP70-1143 and the older cultivar

673 R570. For SP80-3280, apart from changes compatible with transcript editing there were no differences

674 between our genomic and transcriptomic assemblies. Comparisons were also made to the LCP95-384,

675 RB72454 and Q165 sugarcane chloroplasts that we had previously assembled (Lloyd Evans \& Joshi,

676 2016), as well as the Q155 (GenBank: NC_029221) (Hoang et al., 2016), NCo310 (GenBank:

677 NC_006084) (Asano et al., 2004) and RB867515 (GenBank: KX507245) (Barbosa et al. 2016)

678 assemblies from GenBank.

\section{Transcriptomic Coverage of Multiple-chromosome Mitogenomes}

Mapping of transcriptomic data from 18 sugarcane RNA-seq datasets to the SP80-3280 assembly

681

682

683

684

685

686

687

688 revealed that the mitogenome of sugarcane is completely transcribed (Figure 7). We observed a mix of processed (spliced) and unspliced transcripts, with $99.995 \%$ of the mitochondrial chromosomes covered by sequence (i.e. not Ns). Only in a single instance, were all mapped transcripts processed. This being the start codon of nad1, where the entire set of DNA reads had cytosine in the first position, whilst all the RNA-seq reads had an Uracil (see Figure 7 for the mapping data). Moreover, there was complete coverage of the SP80-3280 chloroplast by transcriptomic data.

We also assembled the sugarcane SP80-3280 chloroplast from transcriptomic data. The assembly was the same length as our genomic assembly (Table 4). However, there were 45 sequence substitutions. 
690 and Cucumis sativus also demonstrated complete expression of all mitochondrial chromosomes

691 (Supplemental Document S3).

\section{Expression and Molecular Modelling of Sugarcane rbcL}

Annotation of the sugarcane mitochondrion revealed that sugarcane might, uniquely, possess a

694 functional rbcL molecule in its mitochondrion. The C-terminus of this is different from that of the

695 chloroplast model, but a new stop codon is in frame and the altered amino acids are all within the

696 disordered C-terminus and do not contribute to the functional core of the molecule.

697

Alignment of mitochondrial and chloroplast rbcL proteins (Supplemental Document S4) revealed

698 that the C-terminus of SP80-3280 mitochondrial $\mathrm{rbcL}$ is unique. This allowed expression analysis to be performed just on the mitochondrial copy of the gene. Transcript mapping analysis (Table 5) using read mapping against the unique $\mathrm{C}$-terminal region of the mitochondrial version of rbcL reveals that

701 mitochondrial $\mathrm{rbcL}$ is expressed at a greater level than background (15x up-regulation) though nowhere near the expression of the reference gene (nad6) (181x up-regulation). However, this is sufficient to demonstrate that rbcL is expressed in the mitochondrion.

704 To see if this mitochondrial copy of rbcL might be functional, the protein sequences of the

705 chloroplast and mitochondrial copies of sugarcane $\mathrm{rbcL}$ were modelled by homology with the Phyre ${ }^{2}$ server. In both cases, $>97 \%$ of all residues were modelled with $93 \%$ confidence. The template for modelling was non-activated spinach rubisco in complex with its substrate: ribulose-1,5-bisphosphate (PDB: 1RCX) (Taylor \& Anderson, 1997). To ensure that the initial mapping had not over-constrained the molecules to the same structure molecular dynamics simulations were performed. Superimposing the sugarcane rbcL structures onto the spinach template revealed that all contacts made by spinach rbcL with

711 the substrate are also made by the sugarcane chloroplastic and mitochondrial versions of the rbcL subunit, 712 indicating that sugarcane mitochondrial rbcL could be active and functional. In addition, superposition of 
713 the sugarcane models revealed that they were essentially identical (Figure 8) with a root mean square 714 difference (RMSD) of $0.356 \AA$. 


\section{Discussion}

\section{Mitochondrial Genome Assembly and Annotation}

717 Using Illumina TruSeq Synthetic Long Reads and an iterative approach we were able to assemble

718 the complete mitochondrial genome of sugarcane cultivar SP80-3280. Whilst there were a large number

719 of repeats within each chromosome (Table 4), few repeats were found to be common between both

720 chromosomes. This makes it unlikely that the chromosomes can recombine to form a master circle.

721

Subsequent to our initial assembly of the SP80-3280 mitochondrion, the paper of Shearman et al.,

722 (2016) was published. This revealed an independent assembly of the mitochondrion of a sugarcane

723 hybrid cultivar (Khon Kaen 3). Their chromosome 1 was 300784 bp long and their chromosome 2 was

724144698 bp long. Differences were due to a single deletion in SP80-3280 chromosome 1 and a single

725 insertion in SP80-3280 chromosome 2 (both in AT rich repeat regions). The remainder of the sequence is

726 almost identical. This is hardly surprising, as both cultivars share a (recent) common female parent. As

727 we have the complete mitochondrial sequences of SP80-3280, SP70-1143 and Khon Kaen 3, the

728 mitochondrial genome of SP70-1143 was also assembled so that the relatedness between these three

729 cultivars could be examined.

730 The mitogenomes of sugarcane hybrid cultivars LCP85-384, RB72454 and R570 are more

731 divergent, with chromosome sizes of $300943+144679,300828+144692$ and $300786+144736$,

732 respectively. The main differences being insertions and deletions within AT-rich repeat regions as well

733 as single nucleotide substitutions distributed throughout the genome (Table 4).

734 The assembly of $S$. officinarum IJ76-514 proved to be more interesting. A previous study, using

735 BLAST to map IJ75-514 reads to a sugarcane mitochondrial genome assembly revealed very few

736 differences between the accessions (Shearman et al., 2016). To see if this was the case, we employed a

737 more systematic assembly approach, attempting to assembly the mitogenome of S. officinarum IJ76-514

Peer) reviewing PDF | (2019:02:35349:1:1:NEW 5 Jul 2019) 
738 from scratch. Chromosome 1 was assembled with only 6 small gaps, but chromosome 2 had significant

739 gaps that could not be closed. Indeed, base-by-base comparisons of the SP80-3280 assemblies and the

740 IJ76-514 assemblies revealed a total of 1102 sequence variations (Table 4).

741 Illunina-based assembly of the mitochondrial genome of the sugarcane cultivar SP80-3280

742 identified two mitochondrial chromosomes with no obvious master circle. However, Unicycler assembly

743 for the same synthetic long reads (Figure 2a) yielded an almost completely resolved graph, but with five

744 potential join poinys between the two mitochondrial chromosomes. However, as the potential merge

745 points $(\mathrm{p} 1=296 \mathrm{bp}, \mathrm{p} 2=177 \mathrm{bp}, \mathrm{p} 3=188 \mathrm{bp}, \mathrm{p} 4=188 \mathrm{bp}, \mathrm{p} 5=176 \mathrm{bp})$ are all shorter than the read

746 length (ranging from 101bp in SP80-3280 to 152bp in LCP85-384) no single read can span them, thus

747 the joins could be spurious. This also highlights also a potential problem with Illumina's synthetic long

748 read technology as these long reads are assembled from short reads. Indeed, a synthetic long read only

749 assembly provides exactly the same assembly graph as the short read only data (Figure 2a).

In contrast, assembly of the R570 sugarcane cultivar mitochondrial genome from PacBio Sequel

751 long reads yieldes an assembly graph with two isolated mitochondrial chromosomes and no reads joining

752 them (Figure 2b). Moreover, when all possible paths through the potential merge poinst (Figure 2a) were

753 extracted as sequence and the full R570 PacBio reads set were mapped to them no single read supported

754 the existence of a mastercircle. This is in agreement with the findings of Shearman et al. (2016), though

755 our analysis of R570 had over 100x more coverage.

756 Gene and transcript mapping analyses across the five potential join points between the two

757 mitochromosomes revealed that $\mathrm{p} 1$ was overlapped by the mitochondrial gene rps 4 on chromosome 2,

$758 \mathrm{p} 4$ was overlapped by ccmFc on chromosome 1 and 55 was overlapped by nad6 on chromosome 2 .

759 Transcript mapping revealed a novel sequence supported by 50 transcriptomic long reads covering $\mathrm{p} 4$ on

760 chromosome 1 . The three genes, $\mathrm{rps} 4, \mathrm{ccmFc}$ and nad6 are essential for mitochondrial function.

761 Disruption of these four genes by the formation of a master circle, would lead the mitochondrion to be 
762 non-functional. The transcript covering $\mathrm{p} 4$ on chromosome 1 may be a novel CMS factor, the expression

763 of which is confirmed by transcript analyses (Supplemental Document S2). This leaves only two

764 potential join points between mitochondrial chromosomes 1 and 2.

To further exclude the presence of a master circle, capture primers were designed against two

766 conserved genes in both mitochondrial chromosomes 1 and 2. Additional primers were generated to

767 capture reads corresponding to potential master circle sequences across $\mathrm{p} 2$ and $\mathrm{p} 3$ conserved sequences.

768 Primers for the individual mitochondrial chromosomes successfully captured and enriched mitochondrial

769 DNA. No DNA could be detected from capture by master circle specific primers. However, all samples

770 were combined for library preparation and ONT MinION sequencing. If the master circle exists, the

771 standard mitochondrial chromosome capture would capture it and the master circle specific primers

772 would enrich for it. Assembly of the MinION reads yielded two completely distinct chromosomes (Figure

773 2c) with no long reads connecting them. Thus, both PacBio Sequel reads for the cultivar R570 and

774 MinION sequencing of captured mitochondrial chromosomes for the cultivar SP80-3280 show no support

775 for the existence of a sugarcane mitochondrial master circle. The possibility of master circles bing formed

776 from very short repeats is excluded by Illumina short read data as no short reads were found that joined

777 the two sugarcane mitochondrial chromosomes.

In fact, if the master circle exists then it must be identified by sequencing and mitochondrial

779 isolation - particularly from developing tissue that replicates rapidly. However, our assemblies from

780 both high depth PacBio and sequence capture based ONT data shows no sign of a master circle (this

781 confirms the findings of Shearman et al. (2016)). Though assembly graphs from Illumina short read data

782 indicates five putative conserved sequences between mitochondrial chromosomes 1 and 2 - existence of

783 a master circle is not supported by long read data (Figure 2). These findings cast some doubt on the

784 veracity of master circles assembled from Illumina data alone, as inter-chromosomal joins, if the

785 conserved regions are longer than the base read length, could be artifactual. Although short conserved

786 regions between the two chromosomes of the sugarcane mitochondrial chloroplasts (Table 4) could result 
787 in a master circle being formed, assembly using Illumina, PacBio and MinION data do not support this -

788 as there are no reads that link both chromosomes together. Thus, in none of our analyses did we identify

789 any sequence reads that could link the two sugarcane mitochondrial chromosomes together to form a

790 master circle. This places sugarcane amongst the few plant species with no mitochondrial master circle,

791 the other examples being Cucumis sativus (Alverson et al., 2011) and Silene spp (Sloan, 2013).

792

In all the sugarcane cultivars assembled, two long repeats exist in chromosome 1 . This leads to

793 the intriguing possibility that chromosome 1 could have master circle like properties and might exist in

794 multiple conformations. As reported by Shearman et al. (2016) a single alternate arrangement was

795 identified for chromosome 1 in SP80-3280 and R570 data that involves the 4kbp inverted repeat that

796 occurs within this chromosome, with long reads spanning both copies. The alternate arrangement results

797 in an inversion of the $120 \mathrm{kbp}$ segment between the two repeats and deletion of one of the inverted repeats

798 with $3 / 8$ of the reads supporting the inversion versus $5 / 8$ of the reads supporting the arrangement as

799 presented in Figure 2.

800

To test whether mitochondrial chromosome 1 might have master circle-like properties, capture

801 primers were designed for the $4 \mathrm{kbp}$ inverted repeat region. Sequencing and assembly of the isolated

802 mitochondrial DNA revealed predominantly the assembly as in Figure 2. However, a total of 93 long

803 reads supported the separation of mitochondrial chromosome 1 into two sub-genomic circles centred

804 around the 4kbp repeat, as shown in Figure 2c. Thus, though a rare event mitochondrial chromosome 1 in

805 sugarcane hybrids can be present as subgenomic circles, with mt1 itself acting as a kind of master circle.

806 Thus, though we have evidence for the presence of two alternative conformations of sugarcane

807 mitochondrial chromosome 1 (one of which is very rare in sequence space), despite exhaustive analyses,

808 including sequence capture and long read assembly we find no evidence for the presence of a

809 mitochondrial master circle in sugarcane.

PeerJ reviewing PDF | (2019:02:35349:1:1:NEW 5 Jul 2019) 


\section{Phylogenenomic Analyses}

811 The mitochondrial phylogeny (Figure 3) shows the expected topology, as described previously

812 (Lloyd Evans, Joshi \& Wang 2019) with Sorghum bicolor as the outgroup. Miscanthus is 4.3 million

813 years divergent from sugarcane with S. spontaneum 1.37 million years divergent. Saccharum officinarum

814 diverged 590000 years ago from the lineage of modern sugarcane hybrid cultivars. This confirms our

815 previous findings (Lloyd Evans \& Joshi, 2016), demonstrating that the lineage leading to modern

816 sugarcane hybrid cultivars is a separate species (Saccharum cultum Lloyd Evans and Joshi) from

817 Saccharum officinarum. The dating of the separation of genus Saccharum from Miscanthus at 4.3

818 million years and S. spontaneum from the other Saccharum species at 1.37 million years is in good

819 agreement (3.8 million years and 1.4 million years) with our previous study (Lloyd Evans \& Joshi, 2016).

820

821

As expected, SP70-1143 emerges as ancestral to both SP80-3280 and Khon Kaen 3 (Figure 3),

822 confirming the shared parentage of these three cultivars. Interestingly, R570 emerges as being ancestral

823 to the SP (São Paolo, Brazil) cultivars, representing the first placement of this cultivar in any

824 phylogenetic analysis.

825 The presence of indels and sequence variants within the mitochondrial genomes of sugarcane

826 cultivars, even when they share a recent common female ancestor, indicates that mitogenomes could be

827 the sequence of choice for analyzing the relationships between closely related cultivars. However, our

828 data demonstrate that complete (or very near complete) mitochondrial genomes need to be used for this

829 type of analysis. Potentially, this could work well within the sugarcane cultivar collection, as they are

830 likely to be closely related sequences and phylogenetic confusion due to cross-over with the nuclear

831 genome will be minimal. As with all phylogenetic/phylogenomic analyses the main issue is that of

832 obtaining a meaningful outgroup. However, the approach undertaken in this paper of mapping to a

833 reference genome prior to alignment shows a way forward. Indeed, our partial alignment of a Miscanthus

PeerJ reviewing PDF | (2019:02:35349:1:1:NEW 5 Jul 2019) 
834 mitogenome to the sugarcane reference would make an ideal outgroup for such an analysis.

\section{Transcriptomic Read Mapping}

836

For the first time we have mapped genome-scale transcriptomic reads to a complex (multi-

837 chromosome) plant mitochondrial genome. The majority of spliced reads are between the start of

838 mitochondrial chromosome 1 and the end of mitochondrial chromosome 2 (shown boxed in Figure 4).

839 Thus it appears that the two chromosomes of the sugarcane mitochondrial genome are combined at the

840 spliceosomal level. Indeed, of the 111 significant splicing events identified (Supplemental Table S3) 23

841 (20.7\%) were between the two mitochondrial chromosomes. Unlike in eukaryotic genomes, splice sites

842 were clustered at genomic loci (Figure 4, Supplemental Table S3) and almost 50\% of splice sites were

843 within coding regions. Recently (Tsujimura et al., 2018) reported on the three mitochondrial

844 chromosomes of Allium cepa (onion) CMS line Momiji-3. However, unlike in sugarcane the

845 mitochondrial sub-circles of onion can combine into a master circle through recombination at long

846 repeats. Though the authors of the Allium cepa paper mapped transcriptomic reads to the mitogenome,

847 they did not report complete expression and they did not perform spliceosomal analyses. They reported

848 only on RNA editing within the genome, describing 635 editing positions.

849

Unfortunately, there were insufficient polyA baited reads available in NCBI's sequence read

850 archive to analyse the regions that had polyA tails and were programmed for degradation within the

851 sugarcane mitochondrial transcriptome. As a result, polyA baited reads were mapped to the Sorghum

852 bicolor BTx623 mitogenome instead. PolyA reads only covered $18.9 \%$ of the mitochondrial genome.

853 The regions covered are shown in Figure 5 and full details of the regions and the genome annotation

854 associated with them are given in Supplemental Table S4. In all cases, regions covered are secondary

855 copies of mitochondrial genes, individual exons, pseudogenes, genes captured from the chloroplast,

856 repeat regions, introns and intra-genic regions. These are precisely the regions that would be expected to

857 be tagged for degradation in a mitochondrial genome that is completely transcribed.

PeerJ reviewing PDF | (2019:02:35349:1:1:NEW 5 Jul 2019) 


\section{Identification of Possible CMS factors in Sugarcane}

859

Based on mappings to an orthologue of an Oryza rufipogon a novel CMS factor, CMS1 was

860 identified in the sugarcane mitogenomes. Typically such CMS factors are gene fusions and contain a

861 transmembrane domain. At the protein level, the O. rufipogon and sugarcane ORFs differ by 14 internal

862 amino acid substitutions (five of which are functionally synonymous) and the substitution of IleIle in the

863 rice C-terminus of the protein for TyrLysAsn in the sugarcane orthologue's C-terminus. Both proteins

864 have a predicted transmembrane helix (Figure 6) and both proteins are derived from a nad9 precursor in

865 the mitochondrial genome. Indeed, at the DNA sequence level the CMS protein in sugarcane is identical

866 to nad9 in rice except for seven base substitutions. Interestingly, bases 1 to 249 of the sugarcane

867 mitochondrial protein mapped twice to a sugarcane SP80-3280 genomic sequence (NCBI: MF737055).

868 This potential CMS factor was found in all the modern sugarcane hybrid mitochondrial genomes

869 assembled in this study and was also found to be present (but not annotated) in the previously published

870 Khon Kaen 3 mitochondrial genome (Shearman et al., 2016). As a direct orthologue of rice ORF113 it is

871 therefore highly likely that this newly discovered sugarcane mitochondrial open reading frame is a

872 cytoplasmic male sterility factor.

873

Our partial assembly of the $S$. spontaneum mitogenome indicates that the $S$. spontaneum

874 mitochondrion may have undergone re-arrangement only $320 \mathrm{bp}$ upstream of the CMS gene locus. This

875 leads to the intriguing possibility that the CMS factor has been lost and re-gained several times through

876 the evolution of the Andropogoneae. Expression analysis (Supplemental Document S1) demonstrates that

877 this gene is functional and expressed in sugarcane.

PeerJ reviewing PDF | (2019:02:35349:1:1:NEW 5 Jul 2019) 
881 Interpro domain analyses revealed three main domains - an N-terminal intracellular domain, a central

882 transmembrane domain corresponding to the amino terminus of nad6 and a novel C-termnal extracellular

883 domain (Figure 6 and Supplemental Document S2). The N-terminal domain is the normally untranslated

884 start of nad6 in the mitochondrion. A fusion protein such as this, with extension of nad6, the fusion of an

885 electron transport protein to an extracellular domain has all the hallmarks of a CMS protein.

886 A CMS factor based on nad6, whilst not common is not unknown. In the dicot Mimulus guttatus,

887 (now Erythranthe guttata), (seep monkeyflower or common yellow monkeyflower), 3' extensions of the

888 core nad6 gene are associated with cytoplasmic male sterility (Case and Willis 2008) and are rescued by

889 PPR proteins. Nad6, NADH-ubiquinone oxidoreductase chain 6 also possesses two of the main

890 characteristics for a CMS fusion protein in that it is part of the mitochondrial electron transport system

891 and contains a transmembrane domain. It is fused to both an inner membrane domain on the N-terminus

892 and an extracellular domain on the C-terminus in the sugarcane fusion form (Supplemental Document S2).

893 Sequence analysis in Sorghum bicolor demonstrates that apart from 22 nucleotide variants, the genomic

894 version of the complete transcript of the potential CMS factor is identical to the mitochondrial region.

895 This region emerges as one of eight large regions of the sorghum mitogenome transferred from the

896 Sorghum bicolor BTx623 mitochondrial genome to chromosome 9 in the nuclear genome. Thus it seems

897 likely that the nad6 fusion occurred in the mitochondrion first and was subsequently transferred to the

898 nuclear genome. Indeed, when the DNA sequence of the C-terminal end of the protein is isolated and

899 blasted it matches an unannotated region of the Tripsacum dactyloides cv Pete mitogenome.

900 Sequence assembly in a range of species between the Tripsacinae and Sacchainae, with

901 Chrysopogon zizanoides as an outgroup allowed the presence and origin of this novel putative CMS

902 factor to be determined (Figure 9). The gene seems to have arisen as a gene fusion in the common 
903 ancestor of Chrysopogon, the Tripsacinae and Coix. The gene evolved independently in Chrysopogon,

904 though it seems to be expressed (Supplemental Document S2); however it became non-functional in

905 Tripsacum and was lost in Zea mays. An orthologue of the CMS2 gene is present in Coix lacryma-jobi

906 and is present in all species analysed between the core Andropogoneae and Saccharum. Transcriptomic

907 analysis reveals that CMS2 is expressed in sorghum and sugarcane (Supplemental Document S2). The

908 duplication of this mitochondrial region into the genome is held in common between Andropogon

909 virginicus and Sorghum bicolor, further genomic evidence for the separation of Sorghum and Sarga

910 (Lloyd Evans, Joshi \& Wang, 2019).

911 The flip-side of cytoplasmic male sterility is that for pollen viability to be re-gained a restorer

912 factor $(R f)$ gene must be present in the nucleus. Studies on rice reveal three main types of $R f$ genes:

913 Pentatricopeptide-repeat (PPR) proteins (Gaborieau, Brown \& Mireau, 2016), ubiquitin domain proteins

914 (Fujii et al., 2014) and glycine-rich proteins (Itabashi et al., 2011). An example of each was taken from

915 characterized Oryza sativa proteins (genome references: Rf1: Os05g0207200; Ubiquitin domain

916 containing protein Os10g0542200 and Rf2 Os02g0274000) and the Sorghum bicolor BTx623 orthologues

917 were identified. Using these the sugarcane orthologues were assembled using a bait and assemble

918 methodology (Lloyd Evans \& Joshi 2017). Single sugarcane orthologues were obtained for the ubiquitin

919 domain and glycine rich proteins, but multiple PPR proteins were assembled. Typically this is a large

920 gene family in plants, often with 600 or more members (numbers from orthology in the Ensembl sorghum

921 and maize genome data). Two criteria seem to limit functional PPR proteins in CMS. They must contain a

922 suitable number of duplicated PPR domains and must be targeted to the mitochondrion (Schmitz-

923 Linneweber \& Small, 2008). A pipeline was scripted, whereby as many orthologues of the rice PPR

924 protein were assembled as possible. These orthologues were checked for full-length CDSs and the CDSs

925 were translated to protein sequence. The proteins were piped to a local implementation of TPpred2

926 (Savojardo et al., 2014) and MU-LOC (Zhang et al., 2018) to check for a mitochondrial transit peptide. Of

927239 transcripts assembled, only one had a predicted mitochondrial transit peptide and this was taken on

Peer] reviewing PDF | (2019:02:35349:1:1:NEW 5 Jul 2019) 
928 for further analyses and validation by PCR amplification and sequencing. Domain analyses of the two

929 CMS protein and the three restorer proteins are shown schematically in Figure 6.

\section{Sugarcane Mitochondrial Genome Maintainer of Stability Genes}

931 Two genes, RECA1 and RECG helicases (Odahara et al. 2015) are responsible for maintaining

932 mitochondrial genome stability by suppressing gross rearrangements induced by aberrant recombination

933 between short dispersed repeats. In Physcomitrella patens RECG is targeted towards the mitochondrion

934 and RECA1 is dual targeted towards the chloroplast and mitochondrion. In angiosperms, RECA1 is

935 typically annotated as being targeted towards the chloroplast. The orthologues of these two genes were

936 identified in Zea mays and Sorghum bicolor (using the Ensembl plant genome browser). The sugarcane

937 orthologues were assembled using transcriptomic data for the cultivar SP80-3280. Amplification primers

938 were designed from these assemblies and were employed to amplify the transcripts from SP80-3280 prior

939 to ONT MinION sequencing and Canu assembly. Transcriptomic sequences were translated with

940 ExPASY translate and transit peptide sequences were analyzed. For RECG both Localizer and TPpred 2.0

941 predicted a strong mitochondrial targeted transit peptide (the first forty nucleotides). For RECA1,

942 Localizer predicted the presence of a combination of chloroplast, mitochondrial and nuclear localizing

943 transit peptides, whilst TPpred 2.0 predicted the presence of a mitochondrial transit peptide and TargetP

944 predicted a strong chloroplast transit peptide and a weaker mitochondrial transit peptide. Thus it appears

945 that just as in P. patens both proteins can function within the mitochondrion of sugarcane to stabilize the

946 genome and significantly reduce the possibility of intra- and inter-chromosomal rearrangements due to

947 small repeats.

\section{Chloroplast assembly and analyses}

949 Comparisons of the SP80-3280, SP70-1143, LCP95-384, RB72454, Q165, Q155, NCo310, R570

950 and RB867515 chloroplast genomes (sampling the Louisiana, Brazilian, Australian, Réunionese and

951 South African breeding programmes) revealed that chloroplast assemblies were essentially identical, 
952 with only a few sequence substitutions and insertions/deletions distinguishing chloroplasts from diverse

953 global populations (Table 4). The Saccharum officinarum IJ76-514 chloroplast emerges as an outlier,

954 with 26 substitutions, 2 insertions and 5 deletions as compared with the SP80-3280 chloroplast. This

955 shows that modern sugarcane hybrids are derived from a very limited number of female parents, and the

956 chloroplast genomes are almost clonal. The $S$. officinarum IJ76-514 chloroplast is more divergent,

957 supporting the evolutionary separation of $S$. officinarum from the modern sugarcane hybrid cultivars.

958 Our previous analyses (Lloyd Evans and Joshi, 2016) revealed that within the sugarcane

959 chloroplast genome, a single pseudogene, ACR (ACR-toxin sensitivity gene, that confers toxin

960 sensitivity to Escherichia coli) (Ohtani, Yamamto \& Akimitsu, 2002), is conserved from an ancient

961 translocation event with mitochondrial DNA. However, this gene is now absent from the sugarcane

962 mitogenome. BLAST (Altschul et al., 1990) analyses against a local database of whole and partial plastid

963 sequences reveals that this event occurred in the Petrosaviales (about 120 million years ago (Mennes et

964 al., 2013)) and that ACR has been lost from the mitochondria of true grasses.

\section{Sugarcane Mitochondrial rbcL Analysis and Modelling}

966

Annotation of the sugarcane mitochondrion revealed a potentially functional copy of the

967 chloroplast rbcL molecule. Transcriptomic mapping demonstrated that this gene is expressed and $15 \mathrm{x}$

968 up-regulated over background. However, expression analysis by itself is insufficient to prove that the

969 gene is potentially functional. Molecular modelling revealed that despite containing a modified carboxyl

970 terminal the second copy of $\mathrm{rbcL}$ in the mitochondrion of sugarcane had a conserved fold and conserved

971 active site as compared with the chloroplast version of the gene — and thus was potentially functionally

972 active. Capture of rbcL sequences by the mitochondrion has previously been demonstrated in the

973 Andropogoneae. However, in previous cases where this phenomenon has been noted the rbcL gene has

974 been rendered inactive due to internal frameshifts (Clifton et al., 2004). This is the first instance where a

975 potentially functional rbcL molecule has been reported in a grass mitochondrial genome. This could be

PeerJ reviewing PDF | (2019:02:35349:1:1:NEW 5 Jul 2019) 
976 associated with a relatively recent recombination between the mitochondrial and chloroplast genomes in

977 sugarcane. (For a comparative alignment of rbcL proteins, see Supplemental Document S4). This also

978 has severe consequences for phylogenetic analysis, as rbcL is commonly used as a barcode gene as it is

979 believed to be unique to the chloroplast. This is clearly not the case in certain sugarcane cultivars

980 Transcriptomic Coverage of Multiple-chromosome Mitogenomes

981 Mapping of transcriptomic data to the SP80-3280 assembly revealed that the mitogenome of

982 sugarcane is completely transcribed (Figure 7). It was only recently (Shi et al., 2016; Lima \& Smith,

983 2017) that plant chloroplast genomes and a subset of plant mitochondrial genomes were shown to be

984 fully transcribed, and our findings represent the first report of the full transcription of a multi-

985 chromosomal plant mitochondrial genome.

986

SP80-3280 mitochondrial chromosome 1 had 19 unassigned bases (Ns) divided between four

987

distinct regions of the genome. Mitochondrial chromosome 2 had three unassigned bases divided

988

between three distinct regions of the genome. The chloroplast was $100 \%$ covered by transcriptomic reads.

989

As a result, we are confident in saying that the complete plastome complement of sugarcane is

990

transcribed in its entirety.

991

The SP70-1134 mitochondrial genome, which was assembled from a mix of genomic and

992

transcriptomic data, showed considerable identity to both the Khon Kaen 3 and SP80-3280 genomes (to

993

which it is an ancestor). Comparison with SP80-3280 revealed a total of 118 substitutions in

994 chromosome 1 (of which 55 were compatible with $\mathrm{C} \rightarrow \mathrm{U}$ substitutions characteristic of RNA editing).

995 Chromosome 2 revealed 44 substitutions, 22 of which were consistent with RNA editing.

996

Though it has been demonstrated previously (on a small sample) that relatively small

997

mitogenomes are transcribed in their entirety (Lima \& Smith, 2017) this is the first report of the

998 complete transcription of a multi-chromosomal mitogenome. To demonstrate that the phenomenon is

PeerJ reviewing PDF | (2019:02:35349:1:1:NEW 5 Jul 2019) 
999 universal, transcriptomic short reads were also mapped to the multi-partite mitogenomes of Silene

1000 vulgaris (7 chromosomes), Cucumis sativus (7 chromosomes) and Allium cepa L. (2 chromosomes). In

1001 all cases, even for mitochondrial chromosomes with no coding sequences, there was a minimum of

$100291.74 \%$ coverage (Supplemental Document S1).

1003

When the transcriptomic assembly of the sugarcane SP80-3280 chloroplast was analyzed on a

1004 single base level, 22 of these substitutions proved to be $\mathrm{C} \rightarrow \mathrm{U}$, characteristic of RNA editing. The

1005 remainder of the substitutions were $\mathrm{G} \rightarrow \mathrm{A}$, indicating a second form of RNA editing not previously

1006 described for chloroplasts. As a result, there were no sequence differences between the transcript-

1007 assembled and the genome-assembled chloroplasts of SP80-3280 that could not be accounted for by

1008 RNA editing. This also adds Saccharum hybrids to the list of plants with chloroplasts that have been

1009 demonstrated to be transcribed in their entirety.

\section{A Potential Mechanism for intra-chromosomal trans-splicing}

1011 Our data shows that there is no master circle in the sugarcane mitochondrial genome and that the

1012 two chromosomes assembled in this study are fully independent. If this is the case, spliced transcripts that

1013 cross-chromosomal bounds must be integrated by some means. An answer might come from the

1014 euglenozoan eukaryote Diplonema papillatum (Vlcek et al. 2010). It has a far more complex genome

1015 structure and gene structure than plants, where genome consists of numerous small circular chromosomes

1016 none of which appears to encode a complete gene.

1017 A gene's coding sequence is spread out over nine different chromosomes in non-overlapping

1018 pieces (modules), which are transcribed separately and joined to a contiguous mRNA by trans-splicing.

1019 The pattern of splice sites shared between the two mitochondrial chromosomes of sugarcane is compatible

1020 with trans-splicing being the major integrational means for mature transcript assembly. 
1022 together. To test whether this might be a potential scenario in the sugarcane mitogenome a 54bp region

1023 around the cross-chromosomal splice site of nad2 was extracted from the sugarcane mitochondrial

1024 transcriptome. This was blasted against mitochondrial chromosome 1 and chromosome 2. Interestingly,

1025 the 5' end of the sequence (1-28) was found at location 192934-192961 in the SP80-3280 mitochondrial

1026 chromosome 1, whilst the 3' end of the sequence (29-54) was found at location 89613-89588. This raises

1027 the intriguing possibility that splicing within mitochondrial chromosome 1 of sugarcane can create a

1028 guide RNA that would allow splicing across the separate mitochondrial chromosomes of sugarcane. This

1029 would allow for the splicing of individual, distinct mitochromosomal transcripts without a master circle.

1030 Mapping of Illumina reads to this region revealed no reads that joined the two chromosomes, which

1031 excludes the shared sequence between chromosomes 1 and 2 from forming a master circle.

1032 


\section{Conclusion}

1034 We have assembled three sugarcane cultivar mitochondrial genomes from Illumina genomic data, 1035 one from PacBio Sequel long reads and one from ONT MinION long reads. Mapping of transcriptomic 1036 RNA-seq reads to the SP80-3280 mitochondrial genome assembly revealed, for the first time, that the 1037 complete complex mitochondrial genomes of this plant species are transcribed in its entirety, even when 1038 those mitogenomes are sub-divided into distinct chromosomes. Mapping of RNA-seq data to the 1039 sugarcane mitochondrial genomes revealed multiple splice sites, with the major splice species joining 1040 chromosomes 1 and 2 together. Thus the two chromosomes of the sugarcane mitochondrion appear to be 1041 joined at the transcript and not the DNA level. Interestingly, splice sites seem to be distributed into 1042 spliceosomal 'hotspots' with many of these occurring in coding sequences. Moreover, the sugarcane 1043 mitochondrion may be unique amongst plant mitochondria analysed to date in that there are no signs of

1044 repeat or shared sequence between the two mitochondrial chromosomes that would allow recombination 1045 into a master circle (Figure 2). Moreover, the sugarcane gnome contains two genes, RECA1 and RECG

1046 that stabilize the genome, by suppressing gross rearrangements induced by aberrant recombination

1047 between short dispersed repeats. Thus, despite only having two chromosomes the sugarcane

1048 mitochondrion does not fit into the multipartite map mitochondrial genome model. Rather, the sugarcane 1049 mitochondrion appears to be truly multichromosomal (though with only two chromosomes) and these 1050 chromosomes are integrated at the RNA splicing stage, possibly by guide RNA mediated trans-splicing

1052 to the sugarcane cultivar SP80-3280's mitochondrial genome. As a result polyA-baited reads from the 1053 BTx623 cultivar of Sorghum were mapped to the corresponding Sorghum mitogenome. In all cases, the 1054 regions of the sorghum mitogenome covered by polyA reads are exactly those regions that would be 1055 expected to be marked for degradation. This confirms the major bacterial-like role of polyadenylation in 1056 the mitochondrion — that eradicating unwanted transcripts or non-functional by-products of transcript 1057 editing. 

spontaneum and Saccharum officinarum yielded incomplete assemblies demonstrating that sugarcane

1060 hybrids have diverged significantly from all these species. Indeed, when the assembled reads from these 1061 species were mapped to the sugarcane mitochondrial chromosome assembles we were able to use them 1062 to perform a phylogenetic analysis, which revealed the sister relationship of Miscanthus to genus 1063 Saccharum, Saccharum spontaneum to the crown Saccharum species/cultivars and Saccharum 1064 officinarum to Saccharum cultum (the female ancestor of modern sugarcane hybrids).

Sequence level analysis of mitogenomes and chloroplast genomes revealed greater variability in 1066 the mitogenome, indicating that mitochondrial genomes will be of greater utility in determining the 1067 relationships of sugarcane cultivars to each other than chloroplast genomes. Indeed, the lack of 1068 variability amongst chloroplast genomes indicates that modern sugarcane hybrids arose from a very 1069 small pool of S. cultum cytoplasmic donors. Mitochondrial analysis also confirms S. cultum as being 1070 distinct from S. officinarum, adding credence to our previous study (Lloyd Evans \& Joshi, 2016). genome GC contents, meaning that GC content is a viable methodology to distinguish between the three genome types. This is important, as both chloroplast and mitochondrial genomes are transcribed in their entirety, thus it is possible to assemble these plastomes from transcriptomic data (as we have done for both SP80-3280 and SP70-1143 in this study). We also demonstrate that a combination of genomic and

1077 hybrid cultivar SP70-1143). 
1082 cytoplasmic male sterility (CMS), including a CMS gene in the mitochondrial genome and

1083 representatives of the three main restorer-of-function $(R f)$ genes in the nuclear genome. The homology

1084 between ORF113 in O. rufipogon and the potential CMS factor in the sugarcane mitochondrion with

1085 nad9 suggests that this CMS factor may act by affecting complex I (NADH dehydrogenase) of the

1086 electron transfer pathway (Chen et al., 2017). This goes some way to explaining the phenomenon of

1087 incomplete pollen infertility in sugarcane and indicates that CMS in sugarcane is only partially restored.

1088 These findings also point the way to generating CMS and restorer lines from sugarcane cultivars, which

1089 would be a major leap for ward for sugarcane breeding.

1090 


\section{Funding}

1092This work was partly funded by the South African Sugarcane Research Institute.

1093

\section{Author Contributions}

1095DLlE and SVJ conceived the chloroplast assembly from genomic data component; DLlE conceived the 1096remaining experimental aspects, designed and performed the experiments. TH performed an initial feature 1097mapping to mitochondrial genomes and DMRP provided the SP80-3280 genomic and transcriptomic 1098sequence data. SVJ and DLIE supervised TH. DLlE developed all software scripts, performed all the 1099analyses, analysed and interpreted the data and wrote the paper. SVJ and DMRP critically proofread the 1100 final draft of the manuscript. All authors reviewed and accepted the final manuscript.

1101

\section{Acknowledgements}

1103We thank CSS, Waterbeach, Cambridge, for providing the Miscanthus sinensis cv Andante sequence data 1104and performing the sequencing. We are grateful to Oxford Nanopore Technologies for support through their 1105 community access programme and the LMB, Cambridge for access to ultracentrifuges. We would also like to 1106thank Dr L Ramnath for the N22 cDNA library and The British Association of Sugar Technologists for 1107SP80-3280 plant material.

1108

\section{Data Availability}

1110All finished assemblies from this study have been deposited in ENA under the project identifiers 1111PRJEB26367 (for mitochondria), PRJEB26685 (for chloroplasts), PRJEB31395 for sugarcane 1112gene/transcript assemblies and PRJEB32995 for CMS region assemblies. Partial assemblies and assemblies 1113based on transcriptomic data or hybrid data along with all alignments and phylogenetic trees (including 
1114partial assemblies) were deposited in the Dryad Digital Repository ( $<$ DOI available on acceptance $>$ ).

1115Computer code developed for this project is available from GitHub: https://github.com/gwydion1/bifo1116scripts.git.

1117

1118 Competing Interests

1119Declarations of interest: none 


\section{References}

1121 Abraham MJ, Murtola T, Schulz R, Páll S, Smith JC, Hess B, Lindahl E. 2015. GROMACS: High

1122 performance molecular simulations though multi-level parallelism from laptop to super computers.

1123 Software X1:19-25.

1124 Adamo A, Pinney JW, Kunova A, Westhead DR, Meyer P. 2008. Heat stress enhances the accumulation 1125 of polyadenylated mitochondrial transcripts in Arabidopsis thaliana. PLoS One, 3:p.e2889.

1126 Afgan E, Baker D, Batut B, Van Den Beek M, Bouvier D, Čech M, Chilton J, Clements D, Coraor N, 1127 Grüning BA, Guerler A. 2018. The Galaxy platform for accessible, reproducible and collaborative 1128 biomedical analyses: 2018 update. Nucleic Acids Research, 46(W1):W537-W544.

1129 Allen JO, Fauron CM, Minx P, Roark L, Oddiraju S, Lin GN, Meyer L, Sun H, Kim K, Wang C, Du, F. 1130 2007. Comparisons among two fertile and three male-sterile mitochondrial genomes of maize. Genetics, $1131 \quad 177: 1173-1192$.

1132 Altschul SF, Gish W, Miller W, Myers EW, Lipman DJ. 1990. Basic local alignment search tool. 1133 Journal of Molecular Biology, 215:403-410.

1134 Altschul SF, Madden TL, Schäffer AA, Zhang J, Zhang Z, Miller W, Lipman DJ. 1997. Gapped BLAST 1135 and PSI-BLAST: a new generation of protein database search programs. Nucleic Acids Research. $1136 \quad 25: 3389-3402$.

1137 Alverson AJ, Wei X, Rice DW, Stern DB, Barry K, Palmer JD. 2010. Insights into the evolution of 1138 mitochondrial genome size from complete sequences of Citrullus lanatus and Cucurbita pepo 1139 (Cucurbitaceae). Molecular Biology and Evolution, 27:1436-1448.

1140 Alverson AJ, Rice DW, Dickinson S, Barry K, Palmer JD. 2011. Origins and recombination of the 1141 bacterial-sized multichromosomal mitochondrial genome of cucumber. The Plant Cell, 23:2499-2513. 
1142 Arrieta-Montiel MP, Mackenzie SA. 2011. Plant mitochondrial genomes and recombination. In Plant 1143 mitochondria (pp. 65-82). Springer, New York, NY.

1144 Artschwager E, Brandes EW. 1958. Sugarcane (Saccharum officinarum L.) Agriculture Handbook No 1145 122. United States Department of Agriculture. Washington D.C.

1146 Asano T, Tsudzuki T, Takahashi S, Shimada H, Kadowaki KI. 2004. Complete nucleotide sequence of 1147 the sugarcane (Saccharum officinarum) chloroplast genome: a comparative analysis of four monocot 1148 chloroplast genomes. DNA Research, 11:93-99.

1149 Backert S, Börner T. 2000. Phage T4-like intermediates of DNA replication and recombination in the 1150 mitochondria of the higher plant Chenopodium album (L.). Current Genetics. 37:304-314. doi:

$1151 \quad 10.1007 / \mathrm{s} 002940050532$.

1152 Backert S, Lynn Nielsen B, Börner T. 1997. The mystery of the rings: structure and replication of 1153 mitochondrial genomes from higher plants. Trends in Plant Science. 2:477-483. doi: 10.1016/s13601154 1385(97)01148-5.

1155 Bankevich A, Nurk S, Antipov D, Gurevich AA, Dvorkin M, Kulikov AS, Lesin VM, Nikolenko 1156 SI, Pham S, Prjibelski AD, Pyshkin AV. 2012. SPAdes: A New Genome Assembly Algorithm and Its 1157 Applications to Single-Cell Sequencing. Journal of Computational Biology, 19:455-477.

1158 doi:10.1089/cmb.2012.0021

1159 Barbosa MHP, Vidigal PMP, Coelho ASG, Peternelli LA, Novaes E. 2016. Complete chloroplast genome 1160 sequence and annotation of the Saccharum hybrid cultivar RB867515. Genome Announcements.

$1161 \quad 4: \mathrm{e} 01157-16$

1162 Berkman PJ, Bundock PC, Casu RE, Henry RJ, Rae AL, Aitken KS. 2014. A survey sequence 1163 comparison of Saccharum genotypes reveals allelic diversity differences. Tropical Plant Biology. 7:71116483. 
1165 Berthelier J, Casse N, Daccord N, Jamilloux V, Saint-Jean B, Carrier G. 2018. A transposable element

1166 annotation pipeline and expression analysis reveal potentially active elements in the microalga

1167 Tisochrysis lutea. BMC Genomics, 19:378.

1168 Bottino MC, Rosario S, Grativol C, Thiebaut F, Rojas CA, Farrineli L, Hemerly AS, Ferreira PCG. 2013.

1169 High-throughput sequencing of small RNA transcriptome reveals salt stress regulated microRNAs in

1170 sugarcane. PloS One, 8:p.e59423.

1171 Brickell CD, Baum BR, Hetterscheid WL, Leslie AC, McNeill J, Trehane P, Vrugtman F, Wiersema JH.

1172 (eds). 2009. International Code of Nomenclature for Cultivated Plants (ICNCP or Cultivated Plant Code)

1173 incorporating the Rules and Recommendations for naming plants in cultivation. 8th ed., adopted by the

1174 International Union of Biological Sciences International Commission for the Nomenclature of Cultivated

1175 Plants. Scripta Horticulturae. International Society of Horticultural Science. 10:1-184. ISBN 978-90-

1176 6605-662-6.

1177 Brühl R. 2015. A Mac OS X application for 2D-plots from ASCII data. http://rbruehl.macbay.de/.

1178 Accessed 22 February 2019.

1179 Bullerwell CE, Gray MW. 2004. Evolution of the mitochondrial genome: protist connections to animals, 1180 fungi and plants. Current Opinion in Microbiology. 7:528-534.

1181 Calsa Jr T, Carraro DM, Benatti MR, Barbosa AC, Kitajima JP, Carrer H. 2004. Structural features and

1182 transcript-editing analysis of sugarcane (Saccharum officinarum L.) chloroplast genome. Current

1183 Genetics. 46:366-373.

1184 Carlsson J, Leino M, Sohlberg J, Sundstrom JF, Glimelius, K. 2008. Mitochondrial regulation of flower 1185 development. Mitochondrion 8: 74-86. 
1186 Case AL, Willis JH. 2008. Hybrid male sterility in Mimulus (Phrymaceae) is associated with a

1187 geographically restricted mitochondrial rearrangement. Evolution: International Journal of Organic

1188 Evolution, 62:1026-1039.

1189 Chakrabarty D, Chauhan PS, Chauhan AS, Indoliya Y, Lavania UC, Nautiyal CS. 2015. De novo

1190 assembly and characterization of root transcriptome in two distinct morphotypes of vetiver, Chrysopogon

1191 zizaniodes (L.) Roberty. Scientific Reports, 5:18630.

1192 Chevreux B, Wetter T, Suhai S. 1999. Genome sequence assembly using trace signals and additional

1193 sequence information. In: German Conference on Bioinformatics 99:45-56.

1194 Camacho C, Coulouris G, Avagyan V, Ma N, Papadopoulos J, Bealer K, Madden TL. 2008. BLAST+:

1195 architecture and applications. BMC Bioinformatics. 10:421.

1196 Chang S, Yang T, Du T, Huang Y, Chen J, Yan J, He J, Guan R. 2011. Mitochondrial genome

1197 sequencing helps show the evolutionary mechanism of mitochondrial genome formation in Brassica.

1198 BMC Genomics. 12:497.

1199 Chen Z, Zhao N, Li S, Grover CE, Nie H, Wendel JF, Hua J. 2017. Plant Mitochondrial Genome

1200 Evolutionand Cytoplasmic Male Sterility, Critical Reviews in Plant Sciences, 36:55-69. DOI:

$1201 \quad 10.1080 / 07352689.2017 .1327762$

1202 Christensen AC. 2013. Plant mitochondrial genome evolution can be explained by DNA repair

1203 mechanisms. Genome Biology and Evolution, 5:1079-1086.

1204 Clayton WD, Vorontsova MS, Harman KT, Williamson H. (2006 onwards). GrassBase — The Online

1205 World Grass Flora. http://www.kew.org/data/grasses-db.html. [accessed 10 May 2018; 15:30 GMT]

PeerJ reviewing PDF | (2019:02:35349:1:1:NEW 5 Jul 2019) 
1206 Clifton SW, Minx P, Fauron CMR, Gibson M Allen JO, Sun H, Thompson M, Barbazuk WB, Kanuganti

1207 S, Tayloe C, Meyer L. 2004. Sequence and comparative analysis of the maize NB mitochondrial genome.

1208 Plant Physiology, 136:3486-3503.

1209 Conant GC, Wolfe KH. 2008. GenomeVx: simple web-based creation of editable circular chromosome

1210 maps. Bioinformatics, 24:861-862

1211 Cupp JD, Nielsen BL. 2014. Minireview: DNA replication in plant mitochondria. Mitochondrion, 1212 19:231-237. doi: 10.1016/j.mito.2014.03.008.

1213 Daniels J, Roach BT. 1987. Taxonomy and evolution. In: D. J. Heinz Ed. Sugarcane Improvement 1214 Through Breeding. Elsevier, The Hague. pp. 7-84.

1215 Darling AC, Mau B, Blattner FR, Perna NT. 2004. Mauve: multiple alignment of conserved genomic 1216 sequence with rearrangements. Genome Research. 14:1394-1403.

1217 Darriba D, Taboada GL, Doallo R, Posada D. 2012. jModelTest 2: more models, new heuristics and 1218 parallel computing. Nature Methods, 9:772.

1219 de Haas JM, Hille J, Kors F, van der Meer B, Kool AJ, Folkerts O, Nijkamp HJ. 1991. Two potential 1220 Petunia hybrida mitochondrial DNA replication origins show structural and in vitro functional homology 1221 with the animal mitochondrial DNA heavy and light strand replication origins. Current Genetics. $1222 \quad 20: 503-513$.

1223 Drummond AJ, Ho SYW, Phillips MJ, Rambaut A. 2006. Relaxed phylogenetics and dating with 1224 confidence. PLoS Biol. 4:e88.

1225 Drummond AJ, Suchard MA, Xie D, Rambaut A. 2012. Bayesian phylogenetics with BEAUti and the 1226 BEAST 1.7. Molecular Biology and Evolution, 29:1969-1973.

1227 Eddy SR. 1998. Profile hidden Markov models. Bioinformatics, 14:755-763.

Peer] reviewing PDF | (2019:02:35349:1:1:NEW 5 Jul 2019) 
1228 Emanuelsson O, Brunak S, Von Heijne G, Nielsen H. 2007. Locating proteins in the cell using TargetP,

1229 SignalP and related tools. Nature Protocols, 2:953.

1230 Firtina C, Kim JS, Alser M, Cali DS, Cicek AE, Alkan C, Mutlu O. 2019. Apollo: A Sequencing-

1231 Technology-Independent, Scalable, and Accurate Assembly Polishing Algorithm. arXiv preprint

1232 arXiv:1902.04341.

1233 Fujii S, Kazama T, Ito Y, Kojima S, Toriyama K. 2014. A candidate factor that interacts with RF2, a

1234 restorer of fertility of Lead rice-type cytoplasmic male sterility in rice. Rice, 7:21.

1235 Gaborieau L, Brown GG, Mireau H. 2016. The propensity of pentatricopeptide repeat genes to evolve

1236 into restorers of cytoplasmic male sterility. Frontiers in Plant Science, 7:1816.

1237 Gagliardi D, Stepien PP, Temperley RJ, Lightowlers RN, Chrzanowska-Lightowlers ZM. 2004.

1238 Messenger RNA stability in mitochondria: different means to an end. Trends in Genetics, 20:260-267.

1239 Galtier N. 2011. The intriguing evolutionary dynamics of plant mitochondrial DNA. BMC Biology. 9:61.

1240 Goodstein DM, Shu S, Howson R, Neupane R, Hayes RD, Fazo J, Mitros T, Dirks W, Hellsten U,

1241 Putnam N, Rokhsar DS (2011) Phytozome: a comparative platform for green plant genomics. Nucleic

1242 Acids Research. 40:D1178-D1186.

1243 Grativol C, Regulski M, Bertalan M, McCombie WR, Silva FR, Zerlotini Neto A, Vicentini R, Farinelli

1244 L, Hemerly AS, Martienssen RA, Ferreira PCG. 2014. Sugarcane genome sequencing by methylation

1245 filtration provides tools for genomic research in the genus Saccharum. The Plant Journal, 79:162-172.

1246 Gualberto JM, Mileshina D, Wallet C, Niazi AK, Weber-Lotfi F, Dietrich A. 2014. The plant

1247 mitochondrial genome: dynamics and maintenance. Biochimie, 100:107-120.

1248 Gualberto JM, Newton KJ. 2017. Plant mitochondrial genomes: dynamics and mechanisms of mutation.

1249 Annual Reviews of Plant Biology. 68:17.1-17.28.

PeerJ reviewing PDF | (2019:02:35349:1:1:NEW 5 Jul 2019) 
1250 Guo W, Grewe F, Fan W, Young GJ, Knoop V, Palmer JD, Mower JP. 2016. Ginkgo and Welwitschia

1251 mitogenomes reveal extreme contrasts in gymnosperm mitochondrial evolution. Molecular Biology and 1252 Evolution. 33:1448-1460.

1253 Hamani K, Giege P. 2014. RNA metabolism in plant mitochondria. Trends in Plant Science. 19:380-389.

1254 Hoang NV, Furtado A, McQualter RB, Henry RJ. 2015. Next generation sequencing of total DNA from 1255 sugarcane provides no evidence for chloroplast heteroplasmy. New Negatives in Plant Science, 1:33-45.

1256 Huang WC, Yu CC, Hu J, Wang LL, Dan ZW, Zhou W, He CL, Zeng YF, Yao GX, Qi JZ, Zhang ZH, 1257 Zhu RS, Chen XF, Zhu YG. 2015. Pentatricopeptide-repeat family protein RF6 functions with hexokinase 12586 to rescue rice cytoplasmic male sterility. Procedings of the National Academy of Sciences U.S.A.

1259 112:14984-14989.

1260 Huang X, Madan, A. CAP3: A DNA Sequence Assembly Program. 1999. Genome Research. 9:868-877.

1261 Hunt M, De Silva N, Otto TD, Parkhill J, Keane JA, Harris SR. 2015. Circlator: automated

1262 circularization of genome assemblies using long sequencing reads. Genome Biology. 16:294.

1263 Igarashi K, Kazama T, Motomura K, Toriyama K. 2012. Whole genomic sequencing of RT98

1264 mitochondria derived from Oryza rufipogon and northern blot analysis to uncover a cytoplasmic male 1265 sterility-associated gene. Plant and cell physiology, 54:237-243.

1266 Irvine JE. 1999. Saccharum species as horticultural classes. Theoretical and Applied Genetics. 98:1861267194.

1268 Itabashi E, Iwata N, Fujii S, Kazama T, Toriyama K, 2011. The fertility restorer gene, Rf2, for Lead Rice1269 type cytoplasmic male sterility of rice encodes a mitochondrial glycine-rich protein. The Plant Journal, $1270 \quad 65: 359-367$. 
1271 Kelley LA, Mezulis S, Yates CM, Wass MN, Sternberg MJ. 2015. The Phyre² web portal for protein

1272 modeling, prediction and analysis. Nature Protocols, 10:845.

1273 Kersey PJ, Allen JE, Allot A, Barba M, Boddu S, Bolt BJ, Carvalho-Silva D, Christensen M, Davis P,

1274 Grabmueller C, Kumar N. 2017. Ensembl Genomes 2018: an integrated omics infrastructure for non-

1275 vertebrate species. Nucleic Acids Research 46:D802-D808.

1276 Kim D, Langmead B, Salzberg, SL. 2015. HISAT: a fast spliced aligner with low memory requirements.

1277 Nature Methods. 12:357-360.

1278 Kohany O, Gentles AJ, Hankus L and Jurka J. 2006. Annotation, submission and screening of repetitive

1279 elements in Repbase: RepbaseSubmitter and Censor. BMC Bioinformatics, 7:474.

1280 Koren S, Walenz BP, Berlin K, Miller JR, Bergman NH and Phillippy AM. 2017. Canu: scalable and

1281 accurate long-read assembly via adaptive k-mer weighting and repeat separation. Genome Research.

$1282 \quad 27: 722-736$

1283 Krishnan NM, Rao BJ. 2009. A comparative approach to elucidate chloroplast genome replication. BMC

1284 Genomics. 10:237. doi:10.1186/1471-2164-10-237.

1285 Kurtz S, Choudhuri JV, Ohlebusch E, Schleiermacher C, Stoye J, Giegerich R. 2001. REPuter: the

1286 manifold applications of repeat analysis on a genomic scale. Nucleic Acids Research. 29:4633-4642.

1287 Li H. 2018. Minimap2: pairwise alignment for nucleotide sequences. Bioinformatics, 34:3094-3100.

1288 Li H, Durbin R. 2009. Fast and accurate short read alignment with Burrows-Wheeler transform.

1289 Bioinformatics. 25:1754-1760.

1290 Li H, Handsaker B, Wysoker A, Fennell T, Ruan J, Homer N, Marth G, Abecasis G, Durbin R. 2009.

1291 The sequence alignment/map format and SAMtools. Bioinformatics, 25:2078-2079.

Peer] reviewing PDF | (2019:02:35349:1:1:NEW 5 Jul 2019) 
1292 Lima MS, Smith DR. 2017. Pervasive Transcription of Mitochondrial, Plastid, and Nucleomorph

1293 Genomes across Diverse Plastid-Bearing Species. Genome Biology and Evolution. 9:2650-2657.

1294 doi:10.1093/gbe/evx207

1295 Lister DL, Bateman JM, Purton S, Howe CJ. 2003. DNA transfer from chloroplast to nucleus is much 1296 rarer in Chlamydomonas than in tobacco. Gene. 316:33-38.

1297 Liu K, Raghavan S, Nelesen S, Linder CR, Warnow T. 2009. Rapid and accurate large-scale

1298 coestimation of sequence alignments and phylogenetic trees. Science. 324:1561-1564.

1299 Lloyd Evans D, Joshi SV. 2016. Complete chloroplast genomes of Saccharum spontaneum, Saccharum 1300 officinarum and Miscanthus floridulus (Panicoideae: Andropogoneae) reveal the plastid view on 1301 sugarcane origins. Systematics and Biodiversity. 14:548-571.

1302 Lloyd Evans D, Joshi SV. 2017. Herbicide targets and detoxification proteins in sugarcane: from gene 1303 assembly to structure modelling. Genome. 60:601-617.

1304 Lloyd Evans D, Joshi SV, Wang J. 2019. Whole chloroplast genome and gene locus phylogenies reveal 1305 the taxonomic placement and relationship of Tripidium (Panicoideae: Andropogoneae) to 1306 sugarcane. BMC Evolutionary Biology 19:33.

1307 Mackenzie S, McIntosh L. 1999. Higher plant mitochondria. The Plant Cell, 11:571-585.

1308 Manchekar M, Scissum-Gunn K, Song D, Khazi F, McLean SL, Nielsen BL. 2006. DNA recombination 1309 activity in soybean mitochondria. Journal of Molecular Biology. 356:288-299.

1310 Mattiello L, Riaño-Pachón DM, Martins MCM, da Cruz LP, Bassi D, Marchiori PER, Ribeiro RV, Labate 1311 MTV, Labate CA, Menossi M. 2015. Physiological and transcriptional analyses of developmental stages 1312 along sugarcane leaf. BMC Plant Biology, 15:300. 
1313 McKenna A, Hanna M, Banks E, Sivachenko A, Cibulskis K, Kernytsky A, Garimella K, Altshuler D,

1314 Gabriel S, Daly M, DePristo MA. 2010. The Genome Analysis Toolkit: a MapReduce framework for 1315 analyzing next-generation DNA sequencing data. Genome Research. 20:1297-1303.

1316 Mennes CB, Smets EF, Moses SN, Merckx VS. 2013. New insights in the long-debated evolutionary

1317 history of Triuridaceae (Pandanales). Molecular Phylogenetics and Evolution. 69:994-1004.

1318 Mitchell AL, Attwood TK, Babbitt PC, Blum M, Bork P, Bridge A, Brown SD, Chang HY, El-Gebali S, 1319 Fraser MI, Gough J. 2018. InterPro in 2019: improving coverage, classification and access to protein 1320 sequence annotations. Nucleic Acids Research, 47:D351-D360.

1321 Mower JP, Case AL, Floro ER, Willis JH. 2012. Evidence against equimolarity of large repeat 1322 arrangements and a predominant master circle structure of the mitochondrial genome from a 1323 monkeyflower (Mimulus guttatus) lineage with cryptic CMS. Genome Biology and Evolution. 4:670-686.

1324 Nylander JA, Wilgenbusch JC, Warren DL, Swofford DL. 2008. AWTY (are we there yet?): a system 1325 for graphical exploration of MCMC convergence in Bayesian phylogenetics. Bioinformatics. 24:581-583.

1326 Ohyama K, Fukuzawa H, Kohchi T, Shirai H, Sano T, Sano S, Umesono K, Shiki Y, Takeuchi M, Chang 1327 Z, Aota SI. 1986. Chloroplast gene organization deduced from complete sequence of liverwort 1328 Marchantia polymorpha chloroplast DNA. Nature. 322:572-574.

1329 Ohtani K, Yamamoto H. Akimitsu K. 2002. Sensitivity to Alternaria alternata toxin in citrus because of 1330 altered mitochondrial RNA processing. Proceedings of the National Academy of Sciences, 99:2439-2444.

1331 Otto TD, Dillon GP, Degrave WS, Berriman M. (2011). RATT: Rapid Annotation Transfer Tool.

1332 Nucleic Acids Research. doi: 10.1093/nar/gkq1268

1333 Pettersen EF, Goddard TD, Huang CC, Couch GS, Greenblatt DM, Meng EC, Ferrin TE. 2004. UCSF

1334 Chimera - a visualization system for exploratory research and analysis. Journal of Computational

Peer] reviewing PDF | (2019:02:35349:1:1:NEW 5 Jul 2019) 
1335

1336

1337

1338

1339

1340

1341 $1342 \quad 1: 248-258$.

134

134

134

1346

1347

1348

1349

1350

1351

1352

1353

1354

1355 pp.2973-2974.

Chemistry, 25:1605-1612.

Quevillon E, Silventoinen V, Pillai S, Harte N, Mulder N, Apweiler R, Lopez R. 2005. InterProScan: protein domains identifier. Nucleic Acids Research, 33:W116-W120.

Rambaut A, Suchard MA, Xie D, Drummond AJ. 2013. Tracer, Version 1.5. Available at: tree.bio.ed.ac.uk/software/tracer/.

Reddy, B.V., Ramesh, S., Kumar, A.A., Wani, S.P., Ortiz, R., Ceballos, H. and Sreedevi T.K. 2008. Biofuel crops research for energy security and rural development in developing countries. Bioenergy Res.

Riaño-Pachón DM, Mattiello L. 2017. Draft genome sequencing of the sugarcane hybrid SP80-3280. F1000Research, 6: 11859.2. DOI:10.12688/f1000research.11859.2

Rice P, Longden I, Bleasby A. 2000. EMBOSS: The European Molecular Biology Open Software Suite. Trends in Genetics. 16:276-277.

Ronquist F, Huelsenbeck JP 2003. MrBayes 3: Bayesian phylogenetic inference under mixed models. Bioinformatics. 19:1572-1574.

Sanchez-Puerta MV, Garcia LE, Wohlfeiler J, Ceriotti LF. 2017. Unparalleled replacement of native mitochondrial genes by foreign homologs in a holoparasitic plant. New Phytologist. 214: 376-387.

Savojardo, C., Martelli, P.L., Fariselli, P. and Casadio, R., 2014. TPpred2: improving the prediction of mitochondrial targeting peptide cleavage sites by exploiting sequence motifs. Bioinformatics, 30(20),

Schattner P, Brooks AN, Lowe TM. The tRNAscan-SE, snoscan and snoGPS web servers for the detection of tRNAs and snoRNAs. 2005. Nucleic Acids Research. 33:W686-689 .

PeerJ reviewing PDF | (2019:02:35349:1:1:NEW 5 Jul 2019) 
1356 Schmitz-Linneweber C, Small I. 2008. Pentatricopeptide repeat proteins: a socket set for organelle gene 1357 expression. Trends in Plant Science, 13:663-670.

1358 Shearman JR, Sonthirod C, Naktang C, Pootakham W, Yoocha T, Sangsrakru D, Jomchai N,

1359 Tragoonrung S, Tangphatsornruang S. 2016. The two chromosomes of the mitochondrial genome of a

1360 sugarcane cultivar: assembly and recombination analysis using long PacBio reads. Scientific Reports. $13616: 31533$.

1362 Shepard AR, Rae JL. 1997. Magnetic bead capture of cDNAs from double-stranded plasmid cDNA 1363 libraries. Nucleic Acids Research, 25:3478-3480.

1364 Shi C, Wang S, Xia EH, Jiang JJ, Zeng FC, Gao LZ. 2016. Full transcription of the chloroplast genome 1365 in photosynthetic eukaryotes. Scientific Reports. 6:30135.

1366 Slater GS, Birney E. 2005. Automated generation of heuristics for biological sequence comparison. BMC 1367 Bioinformatics. 6:31; doi: 10.1186/1471-2105-6-31

1368 Sloan DB. 2013. One ring to rule them all? Genome sequencing provides new insights into the 'master 1369 circle'model of plant mitochondrial DNA structure. New Phytologist, 200:978-985.

1370 Sloan DB, Alverson AJ, Chuckalovcak JP, Wu M, McCauley DE, Palmer JD, Taylor DR. 2012. Rapid 1371 evolution of enormous, multichromosomal genomes in flowering plant mitochondria with exceptionally 1372 high mutation rates. PLoS Biology. 10:e1001241.

1373 Sobhakumari VP. 2013. New determinations of somatic chromosome number in cultivated and wild 1374 species of Saccharum. Caryologia: International Journal of Cytology, Cytosystematics and Cytogenetics. $137566: 268-274$. 
1376 Sperschneider J, Catanzariti AM, DeBoer K, Petre B, Gardiner DM, Singh KB, Dodds PN, Taylor JM, 1377 2017. LOCALIZER: subcellular localization prediction of both plant and effector proteins in the plant cell. 1378 Scientific Reports, 7:44598.

1379 Stajich JE, Block D, Boulez K, Brenner S, Chervitz S, Dagdigian C, Fuellen G, Gilbert J, Korf I, Lapp H, 1380 Lehväslaiho H, Matsalla C, Mungall CJ, Osborne BI., Pocock MR, Schattner P, Senger M, Stein LD, 1381 Stupka E, Wilkinson MD, Birney E. 2002. The BioPerl Toolkit: Perl Modules for the Life Sciences. 1382 Genome Research. 12:1611-1618. doi:10.1101/gr.361602

1383 Stamatakis A. 2006. RAxML-VI-HPC: Maximum likelihood-based phylogenetic analyses with 1384 thousands of taxa and mixed models. Bioinformatics. 22:2688-2690.

1385 Strehle MM, Purfeerst E, Christensen AC. 2018. A rapid and efficient method for enriching 1386 mitochondrial DNA from plants. Mitochondrial DNA Part B, 3:239-242.

1387 Subramanyam KN, Andal R. 1984. Male Sterility in Sugarcane. Current Science. 53:42-43.

1388 Sukumaran J, Holder MT. 2010. DendroPy: A Python library for phylogenetic

1389 computing. Bioinformatics. 26:1569-1571.

1390 Suzuki H, Yu J, Ness S, O’Connell M, Zhang J. 2013. RNA editing events in mitochondrial genes by 1391 ultra-deep sequencing methods: a comparison of cytoplasmic male sterile, fertile and restored genotypes 1392 in cotton. Mol. Genet. Genomics. 288: 445-457.

1393 Taylor TC, Andersson I. 1997. The structure of the complex between rubisco and its natural substrate 1394 ribulose 1, 5-bisphosphate. Journal of Molecular Biology, 265:432-444.

1395 Teng H, Cao MD, Hall MB, Duarte T, Wang S, Coin LJ. 2018. Chiron: translating nanopore raw signal 1396 directly into nucleotide sequence using deep learning. GigaScience, 7:p.giy037. 
1397 Thorvaldsdóttir H, Robinson, JT, Mesirov JP. 2013. Integrative Genomics Viewer (IGV): high-

1398 performance genomics data visualization and exploration. Briefings in Bioinformatics. 14:178-192.

1399 Toriyama K, Kazama T, Motomura K, Igarishi K. 2013. Rice RT-type Cytoplasmic Male Sterility Causal

1400 Gene and Use Thereof. International Patent Application WO2014027502.

1401 https://patents.google.com/patent/WO2014027502A1/en?oq=Rice+RT-

1402 type + Cytoplasmic + Male + Sterility + Causal+Gene+and + Use+Thereof. + International+Patent + Application

$1403+$ WO2014027502.

1404 Tsujimura M, Kaneko T, Sakamoto T, Kimura S, Shigyo M, Yamagishi M, Toru Terachi T. 2018.

1405 Multichromosomal structure of the onion mitochondrial genome and a transcript analysis. 2018. doi:

1406 10.1016/j.mito.2018.05.001

1407 Turnel M, Otis C, Lemieux C. 2003. The mitochondrial genome of Chara vulgaris: insights into the 1408 mitochondrial DNA architecture of the last common ancestor of green algae and land plants. The Plant 1409 Cell. 15:1888-1903.

1410 Vargas L, Santa Brígida AB, Mota Filho JP, de Carvalho TG, Rojas CA, Vaneechoutte D, Van Bel M, 1411 Farrinelli L, Ferreira PC, Vandepoele K, Hemerly AS. 2014. Drought tolerance conferred to sugarcane by 1412 association with Gluconacetobacter diazotrophicus: a transcriptomic view of hormone pathways. PLoS 1413 One, 9:p.e114744.

1414 Vilella AJ, Severin J, Ureta-Vidal A, Heng L, Durbin R, Birney E. 2009. EnsemblCompara GeneTrees:

1415 Complete, duplication-aware phylogenetic trees in vertebrates. Genome Research. 19:327-335.

1416 Vlcek C, Marande W, Teijeiro S, Lukeš J, Burger G. 2010. Systematically fragmented genes in a 1417 multipartite mitochondrial genome. Nucleic Acids Research, 39:979-988. 
1418 Walker BJ, Abeel T, Shea T, Priest M, Abouelliel A, Sakthikumar S, Cuomo CA, Zeng Q, Wortman J, 1419 Young SK, Earl AM. 2014. Pilon: an integrated tool for comprehensive microbial variant detection and 1420 genome assembly improvement. PloS One. 9:p.e112963.

1421 Wick RR, Judd LM, Gorrie CL, Holt KE. 2017. Unicycler: resolving bacterial genome assemblies from 1422 short and long sequencing reads. PLoS Computational Biology, 13:p.e1005595.

1423 Wick RR, Schultz MB, Zobel J, Holt KE. 2015. Bandage: interactive visualization of de novo genome 1424 assemblies. Bioinformatics, 31:3350-3352.

1425 Zhang, N., Rao, R.S.P., Salvato, F., Havelund, J.F., Møller, I.M., Thelen, J.J. and Xu, D., 2018. MU1426 LOC: a machine-learning method for predicting mitochondrially localized proteins in plants. Frontiers in 1427 plant science, 9.

1428 Zhang T, Hu S, Zhang G, Pan L, Zhang X, Al-Mssallem IS, Yu J. 2012. The organelle genomes of 1429 Hassawi rice (Oryza sativa L.) and its hybrid in Saudi Arabia: genome variation, rearrangement, and 1430 origins. PloS One, 7:p.e42041. 


\section{Figure Legends}

1432 Figure 1. Circular images of the Saccharum hybrid SP80-3280 mitochondrial genome.

1433 Circular diagrams of the mitochondrial chromosomes of sugarcane hybrid cultivar SP80-3280. Bars on 1434 the outer circle represent genes (with forward strand genes on the outer track and reverse strand genes on 1435 the inner track). All genes are labelled and the large direct repeat (DR) and inverted repeats (IR) are 1436 shown and labelled on the centre track of chromosome 1. The inner, grey, circle represents GC content. 1437 Images were drawn with GenomeVX (Conant and Wolfe, 2008).

1439 Figure 2. Assembly graphs for four independent assemblies of the sugarcane mitogenome

1440 Assembly graph images for four independent assemblies of the sugarcane mitogenomes assembly. A)

1441 Assembly graph from the Unicycler assembly of Illumina short reads and Illumina synthetic long reads

1442 for the sugarcane cultivar SP80-3280. Chromosome $1 \mathrm{~s}$ in blue and chromosome 2 is in green. Arrows 1443 show the assembly directions through the two chromosomes. Numbered regions represent putative joins 1444 between the two chromosomes that cannot be resolved by Illumina reads. B) Assembly graph from Canu 1445 assembly of R570 cultivar PacBio Sequel long read data. Both mitochromosomes are fully resolved with 1446 no reads joining them. C) Canu assembly of ONT MinION reads isolated for individual chromosomes 1447 and potential join point between the SP80-3280 chromosomes based on capture primers. Despite an 1448 attempt at enriching for the master circle, it was not found and the mitogenomes assembled as two

1449 chromosomes with no reads joining them. D) Canu assembly of the SP80-3280 mt1 minicircle isolated

1450 by capture primers. Two minicircles are shown, joined by the $4 \mathrm{kbp}$ repeat region in sugarcane

1451 mitochondrial chromosome 1.

1452

PeerJ reviewing PDF | (2019:02:35349:1:1:NEW 5 Jul 2019) 
1453 Figure 3. Phylogram and Chronogram generated from sugarcane mitochondrial chromosome 2 data.

1454 A phylogram (left) was generated from mitochondrial chromosome 2 data for sugarcane and reads

1455 mapped to chromosome 2 for other species. The phylogram was generated with RAxML, and numbers

1456 above nodes represent maximum likelihood bootstrap support, whilst numbers below nodes represent

1457 Bayesian inference support. The scale bar at the bottom represents numbers of substitutions per site. The

$1458 / /$ mark represents long branches that have been reduced by $50 \%$. The image, right, gives a chronogram

1459 generated with BEAST for the mitochondrial data. The scale axis (bottom) gives numbers in millions of

1460 years before present. The numbers at nodes represent the age of the node (in millions of years before

1461 present). Node bars represent 95\% highest probability densities (HPD) on the age of the node.

1462

1463 Figure 4. The Spliceosome of the Sugarcane Mitochondrion

1464 Image of the complete spliceosome of the sugarcane mitochondrion drawn with IGV. Chromosome 1 and 1465 chromosome 2 are concatenated together in this view but the extents of MT1 and MT2 are marked. Both

1466 strands are shown and spliceosomal events occur when the red and blue lines touch the line dividing the

1467 forward and reverse mapped reads. Splice sites typically seem to cluster in hotspots where there is

1468 considerable mapping depth. Though long-range splice events predominate short-range splice events can

1469 still be seen (narrow humps in the background). The most common splice sites (boxed) are between the

1470 start of chromosome 1 and the start of chromosome 2. The denser the colour map the more splice sites 1471 span that region.

1473

1474 Figure 5. PolyA Tailed Mitochondrial RNAs in Sorghum 
1475 An image generated from IGV showing the mapping of polyA-baited transcript reads to the Sorghum

1476 bicolor cv BTx623 mitochondrial genome. Regions of contiguous high mapping depth are boxed and

1477 numbered. A full analysis of the mapped regions, including the genes/features contained therein is

1478 available in Supplemental Table S4.

1479

1480 Figure 6. Domain and protein feature mappings of the sugarcane mitochondrial CMS factor and three 1481 putative genomic restoration factors.

1482 Images represent: A) the sugarcane mitochondrial CMS factor 1, showing the extent of the first, 1483 transmembrane, helix as predicted by TMHMM and the Transmembrane region as predicted by 1484 PHOBIUS as implemented in InterProScan (Quevillon et al., 2005); B) domain schematic for CMS

1485 factor 2, showing a cytosolic domain, a transmembrane domain from nad6 and a C-terminal cytosolic 1486 domain. C) ShRf11, a potential restorer of function 1 like transcript, showing the mitochondrial transit 1487 peptide and all the PPR (pentatricopeptide) repeats within the protein; D) the sugarcane orthologue of 1488 rice and sorghum DSK2 protein, a restorer of function gene with an ubiquitin superfamily domain at the

1489 N-terminus and an UBA-like domain responsible for polypeptide substrate binding at the C-terminus and 1490 E) the ShRf21 (restorer of function 2 like) protein, which has no recognised domains, but which does 1491 contain a conserved glycine-rich region.

1492

1493 Figure 7. Mapping transcriptomic data to the sugarcane SP80-3280 mitochondrion and chloroplast.

1494 Image showing the results of mapping transcriptomic reads to the sugarcane SP80-3280 mitochondrial 1495 and chloroplast genomes. A: SP80-3280 mitochondrial chromosome 1. B: SP80-3280 mitochondrial 1496 chromosome 2. C: SP80-3280 chloroplast genome. The y-axis represents $\log _{10}$ counts for transcript 1497 coverage at each base position within the genome. The x-axis represents base position within the genome. 
1498

1499

1500

1501 Figure 8. Structural comparisons of the sugarcane chloroplast and mitochondrial version of rbcL.

1502 Superimposition and structural comparisons of the sugarcane chloroplast (mauve) and mitochondrial

1503 (green) version of the rbcL (rubisco large subunit). As can be seen, the structures are virtually identical

1504 and apart from truncations in the disordered amino $(\mathrm{N})$ and carboxyl $(\mathrm{C})$ termini of the mitochondrial

1505 protein the only meaningful difference is the prediction of a helix centred on R86 in the chloroplast

1506 molecule and the prediction of a corresponding loop centred on Arg79 in the mitochondrial protein

1507 (shown with an arrow). However, as the sequences in the two regions are identical, this difference is

1508 almost certainly not meaningful. Otherwise, the structures are identical and active site amino acids are

1509 conserved, a strong indication that the sugarcane mitochondrial version of rbcL could be functional.

1510

1511

1512 Figure 9. Origin of the sugarcane CMS2 cytoplasmic male sterility factor.

1513 Image showing the origin of the sugarcane CMS2 cytoplasmic male sterility factor. CMS2 domains are

1514 mapped against a reference tree for the Andropogoneae (derived from Lloyd Evans, Joshi and Wang,

1515 2019). Domains analysed and mapped are: red — nad6 5'; blue nad6 transmembrane region; yellow -

1516 cytoplasmic domain; brown - cytoplasmic domain 3' end. All regions are present in Chrysopogon

1517 zizanoides, but are gradually lost in the Tripsacinae. The full chimeric protein is present in all species

1518 from Coix lacryma-jobi to sugarcane.

PeerJ reviewing PDF | (2019:02:35349:1:1:NEW 5 Jul 2019) 
1519 
1520 Table Legends

1521 Table 1. Species, cultivar data with associated SRA accessions and publications.

1522 A list of sugarcane cultivars, Saccharum species and other species analysed in this study along with the

1523 SRA files used for assembly and mapping and associated publications.

1524

1525

Table 2. Capture primers and flanking sequence employed for mitochondrial isolation.

1526 A list of sugarcane mitochondrial chromosome and bait regions with associated bait primers and 5' and 3'

1527 blocking flanking sequences used to capture different sugarcane mitochondrial conformations prior to 1528 sequencing.

Table 3. Primers used to amplify transcripts from the SP80-3280 and N22 sugarcane cDNA libraries.

1533 A list of primers used to amplify potential restorer of function transcripts in both the SP80-3280 and N22

1534 sugarcane cultivars. This table gives the gene names and types for the three potential CMS restorer of

1535 function transcripts identified in sugarcane. Also given are the forward and reverse primers used to

1536 amplify the transcripts, the length of the amplicons obtained and the melting temperatures $\left(\mathrm{T}_{\mathrm{m}}\right)$ for the

1537 primers. In addition, primers used to amplify the two mitochondrial genome stabilizing factors, RECG

1538 and RECA1 in SP80-3280 are detailed.

1539

1540

1541 Table 4. Comparisons of base-level differences in the mitochondria and chloroplasts of sugarcane

1542 cultivars to the SP80-3280 reference assemblies presented in this paper.

1543 Analysis of base-by base comparisons of several sugarcane mitochondrial and chloroplast assemblies

1544 from different cultivars to the reference SP80-3280 assemblies presented in this paper. Mitochondrial 
1545 data is given at the top and chloroplast data at the bottom. Columns represent: cultivar; total length of

1546 plastome; total number of substitutions; total number of insertions; total number of deletions. For

1547 mitochondria, positions of large direct and inverted repeats and the total number of small repeats are

1548 given. Numbers in brackets give substitutions corrected for transcript post-processing. The label 'gb'

1549 means that the sequence is one downloaded from GenBank.

1550

1551

1552 Table 5. Expression analysis of Mitochonrial rbcL in the sugarcane cultivar SP80-3280.

1553 Analysis of transcript expression for the mitochondrial copy of rbcL in the sugarcane cultivar SP80-3280

1554 compared with nad6, with a random $1000 \mathrm{bp}$ non-coding mitochondrial region used as a reference.

1555 Transcript matches were normalized against gene length and expressed as fold change in comparison to 1556 the non-coding regions.

1557 


\section{Supplemental Materials}

1559

\section{Supplemental Table S1}

1561 A complete listing of splice sites in the sugarcane mitochondrial genome. A listing of all splice sites with 1562 occurrence $>10$ in the sugarcane mitochondrial genome. The table lists the splice sites positions, 1563 upstream and downstream genes and the notes give any coding sequences that the splice sites lie within.

1564

\section{Supplemental Table S2}

1566 Summary of GC content in chloroplast, mitochondrial and nuclear genomes of a representative sample of 1567 Sacchainae. Table headers represent: species, species name; voucher or accession, species voucher, 1568 cultivar name or accession; GenBank accession, GenBank identifier of sequence (if available); reference, 1569 published reference for the sequence and GC Content (\%), percentage GC content in the genome.

1570

\section{Supplemental Table S3}

1572 Table listing polyA baited read mapping to the Sorghum bicolor BTx623 mitochondrial genome. Table 1573 lists the start and end of all contiguous polyA read mapping sites with depth $>500$ reads along with any 1574 genes or notable featured within the mapping region.

1575

1576

1577

\section{Supplemental Table S4}

1579 Transposable elements in the sugarcane SP80-3280 mitochondrial genome. Transposable elements were 1580 derived from the Poaceae dataset of Censor. Column headings: Left and right, position of the 1581 transposable element in the mitochondrial genome (From/To indicates start/end of positions of the 
1582 transposable elements). Orientation: + forward strand; -, complementary. 'Sim' indicates value of

1583 similarity between 2 aligned fragments; 'Pos' is the ratio of positives to alignment length; 'Mm:Ts' is a

1584 ratio of mismatches to transitions in the nucleotide alignment. 'Score', alignment score obtained from

1585 BLAST.

1586

1587

\section{Supplemental Document S1}

1589 Origins and analysis of sugarcane CMS2 transcript and protein. Full analysis of the potential new CMS2 1590 cytoplasmic male sterility factor in sugarcane, including amplification primers for CMS2 mitochondrial 1591 regions, protein alignment comparisons and domain mapping, analysis of domain origins and expression 1592 analyses.

1593

1594 Supplemental Document S2

1595 Assembly graphs for the partial mitochondrial genome assemblies from Saccharum spontaneum

1596 SES234B and Miscanthus sinensis cv Andante showing overlapping BLAST mappings to the SP80-3280 1597 mitochondrial chromosomes.

1598

1599

1600 Supplemental Document S3

1601 Mapping of RNA-seq reads to the multi-chromosomal mitogenomes of three species. Document gives

1602 column graphs of site by site mappings of RNA-seq reads to each chromosome in the multi-chromosome 
1603 mitogenomes of Silene vulgaris, Cucumis sativus and Allium cepa showing almost complete coverage of 1604 each chromosome. The document lists all the SRA datasets employed in each of the mappings.

1605

\section{Supplemental Document S4}

1607 Alignment of the rbcL protein from four sugarcane cultivars in comparison with the equivalent protein 1608 from S. spontaneum, S. officinarum and the sugarcane cultivar SP80-3280 chloroplast genome.

1609 
Figure 1

Circular images of the Saccharum hybrid SP80-3280 mitochondrial genome.

Circular diagrams of the mitochondrial chromosomes of sugarcane hybrid cultivar SP80-3280.

A) Mitochondrial chromosome 1. B) Mitochondrial chromosome 2. Bars on the outer circle represent genes (with forward strand genes on the outer track and reverse strand genes on the inner track). All genes are labelled and the large direct repeat (DR) and inverted repeats (IR) are shown and labelled on the centre track of chromosome 1. The inner, grey, circle represents GC content. Images were drawn with GenomeVX (Conant and Wolfe, 2008).

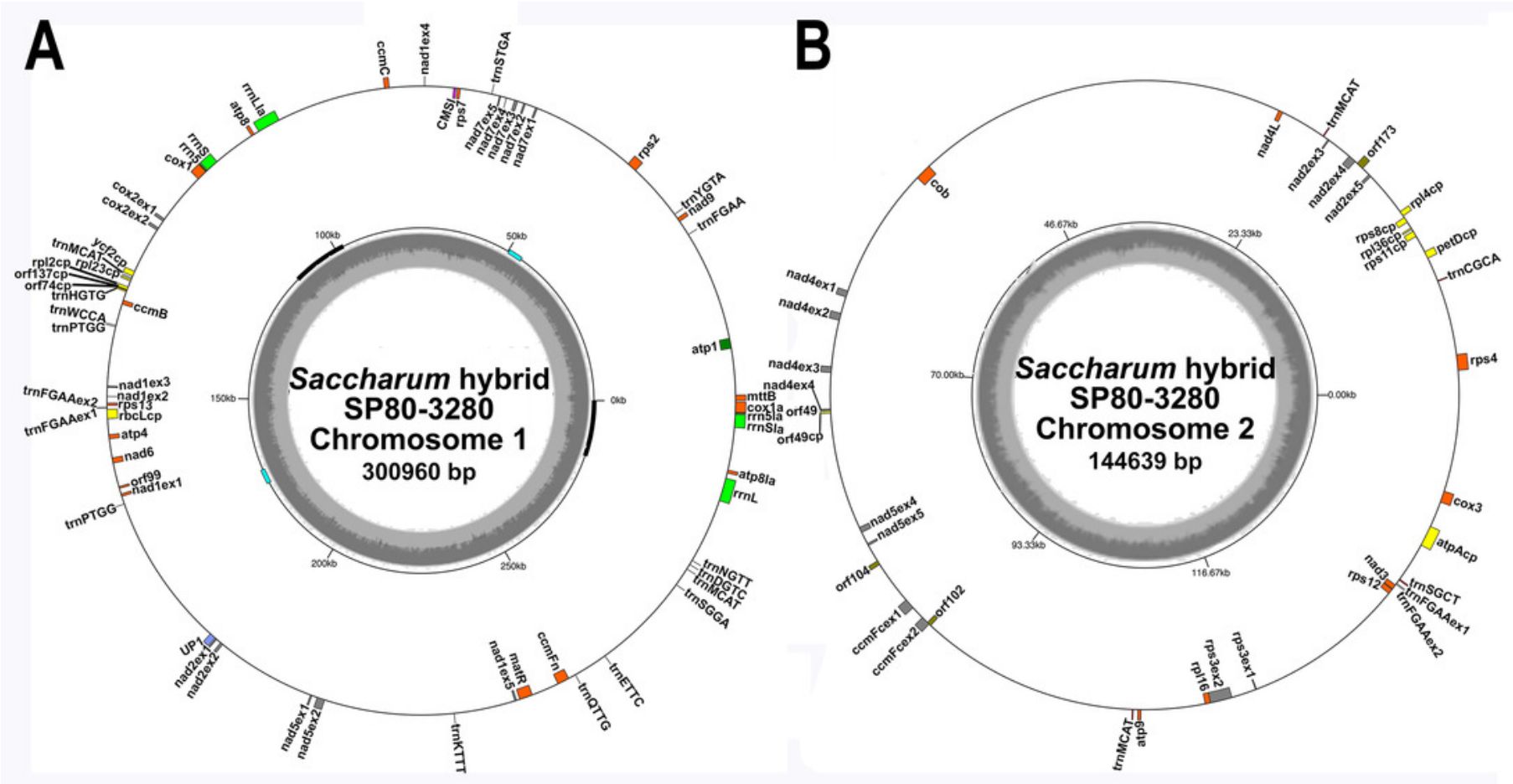




\section{Figure 2}

Assembly graphs for four independent assemblies of the sugarcane mitogenome Assembly graph images for four independent assemblies of the sugarcane mitogenomes assembly. A) Assembly graph from the Unicycler assembly of Illumina short reads and Illumina synthetic long reads for the sugarcane cultivar SP80-3280. Chromosome $1 \mathrm{~s}$ in blue and chromosome 2 is in green. Arrows show the assembly directions through the two chromosomes. Numbered regions represent putative joins between the two chromosomes that cannot be resolved by Illumina reads. B) Assembly graph from Canu assembly of R570 cultivar PacBio Sequel long read data. Both mitochromosomes are fully resolved with no reads joining them. C) Canu assembly of ONT MinION reads isolated for individual chromosomes and potential join point between the SP80-3280 chromosomes based on capture primers. Despite an attempt at enriching for the master circle, it was not found and the mitogenomes assembled as two chromosomes with no reads joining them. D) Canu assembly of the SP80-3280 mt1 minicircle isolated by capture primers. Two minicircles are shown, joined by the $4 \mathrm{kbp}$ repeat region in sugarcane mitochondrial chromosome 1 . 

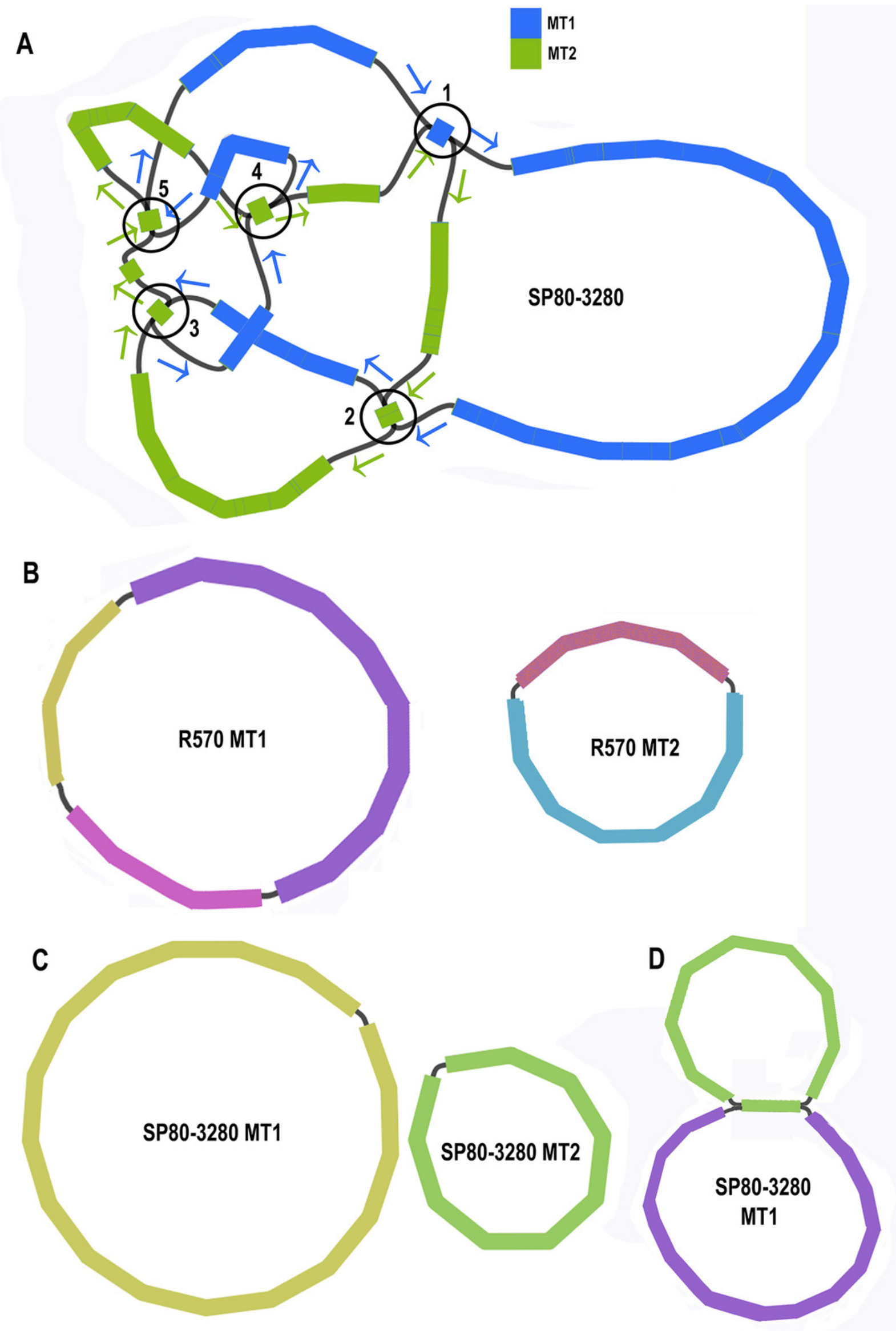


\section{Figure 3}

Phylogram and Chronogram generated from sugarcane mitochondrial chromosome 2 data.

A phylogram (left) was generated from mitochondrial chromosome 2 data for sugarcane and reads mapped to chromosome 2 for other species. The phylogram was generated with RAxML, and numbers above nodes represent maximum likelihood bootstrap support, whilst numbers below nodes represent Bayesian inference support. The scale bar at the bottom represents numbers of substitutions per site. The // mark represents long branches that have been reduced by $50 \%$. The image, right, gives a chronogram generated with BEAST for the mitochondrial data. The scale axis (bottom) gives numbers in millions of years before present. The numbers at nodes represent the age of the node (in millions of years before present). Node bars represent 95\% highest probability densities (HPD) on the age of the node.

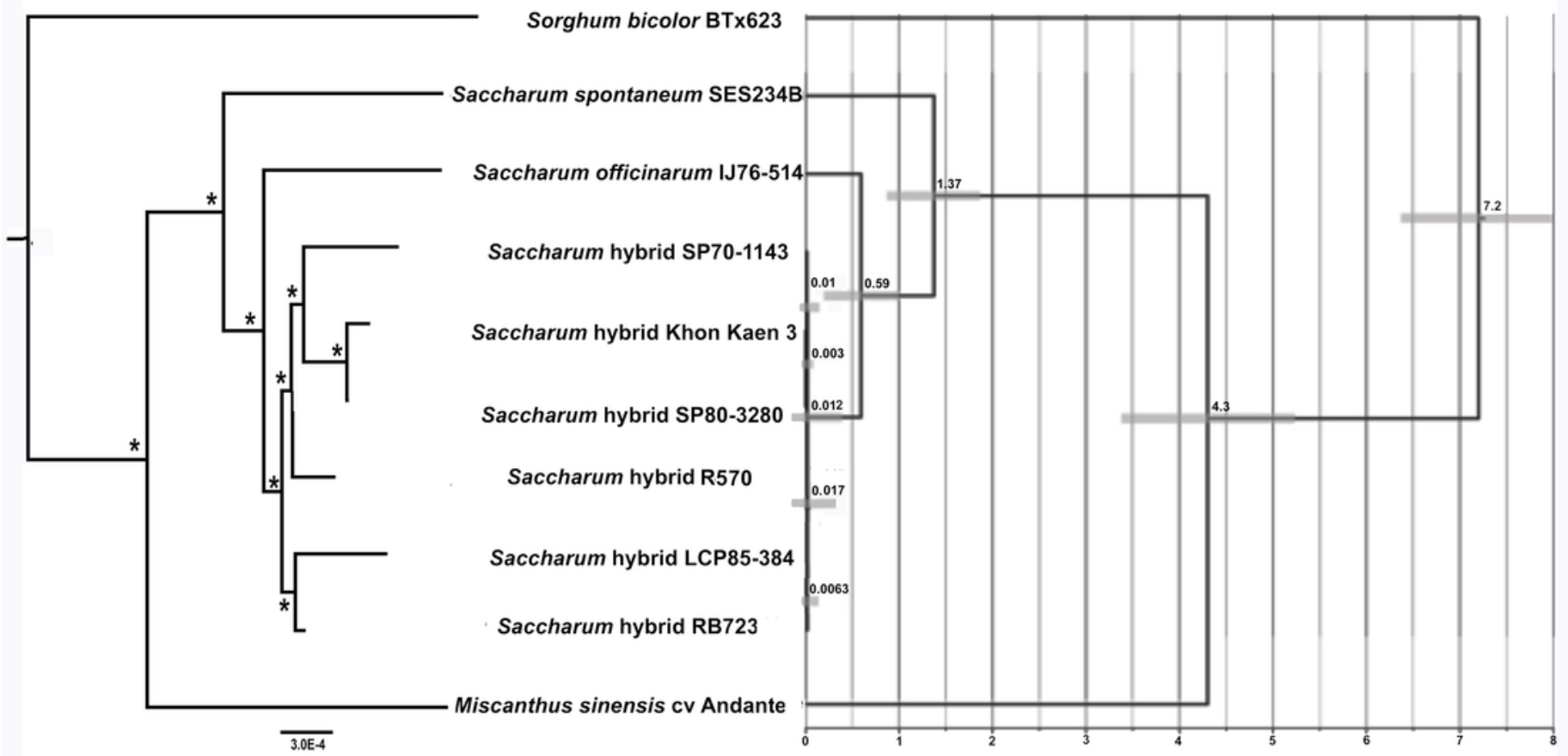




\section{Figure 4}

The Spliceosome of the Sugarcane Mitochondrion

Image of the complete spliceosome of the sugarcane mitochondrion drawn with IGV.

Chromosome 1 and chromosome 2 are concatenated together in this view but the extents of MT1 and MT2 are marked. Both strands are shown and spliceosomal events occur when the red and blue lines touch the line dividing the forward and reverse mapped reads. Splice sites typically seem to cluster in hotspots where there is considerable mapping depth. Though long-range splice events predominate short-range splice events can still be seen (narrow humps in the background). The most common splice sites (boxed) are between the start of chromosome 1 and the start of chromosome 2. The denser the colour map the more splice sites span that region. 


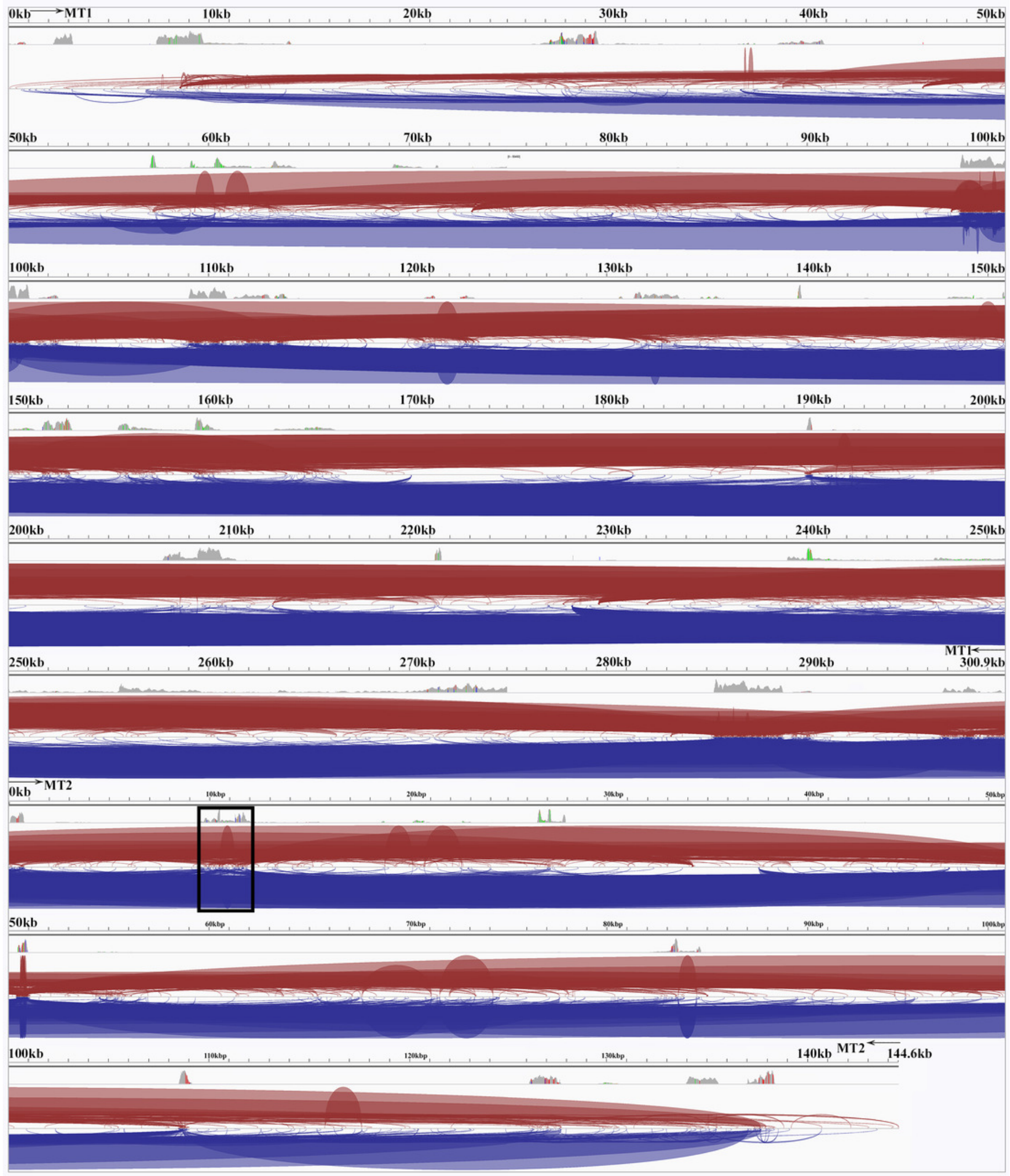


Figure 5

PolyA Tailed Mitochondrial RNAs in Sorghum

An image generated from IGV showing the mapping of polyA-baited transcript reads to the Sorghum bicolor cv BTx623 mitochondrial genome. Regions of contiguous high mapping depth are boxed and numbered. A full analysis of the mapped regions, including the genes/features contained therein is available in Supplemental Table S4.

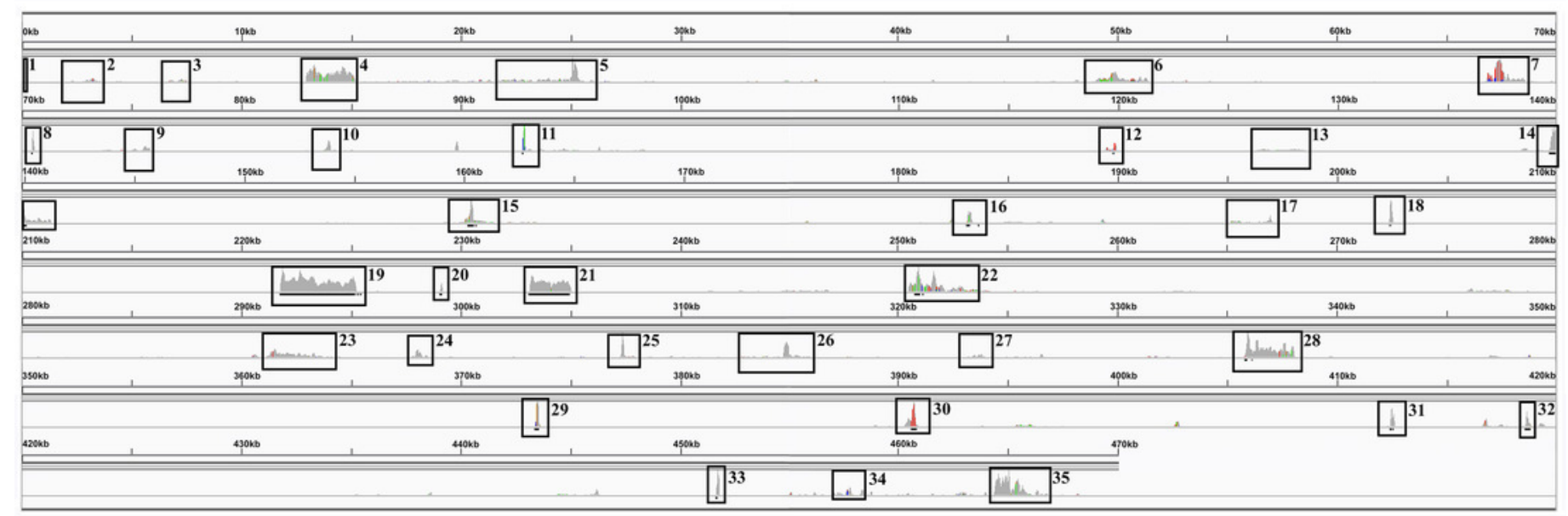




\section{Figure 6}

Domain and protein feature mappings of the sugarcane mitochondrial CMS factor and three putative genomic restoration factors.

Images represent: A) the sugarcane mitochondrial CMS factor 1, showing the extent of the first, transmembrane, helix as predicted by TMHMM and the Transmembrane region as predicted by PHOBIUS as implemented in InterProScan (Quevillon et al., 2005); B) domain schematic for CMS factor 2, showing a cytosolic domain, a transmembrane domain from nad6 and a C-terminal cytosolic domain. C) ShRf1l, a potential restorer of function 1 like transcript, showing the mitochondrial transit peptide and all the PPR (pentatricopeptide) repeats within the protein; D) the sugarcane orthologue of rice and sorghum DSK2 protein, a restorer of function gene with an ubiquitin superfamily domain at the N-terminus and an UBA-like domain responsible for polypeptide substrate binding at the C-terminus and E) the ShRf2I (restorer of function 2 like) protein, which has no recognised domains, but which does contain a conserved glycine-rich region. 


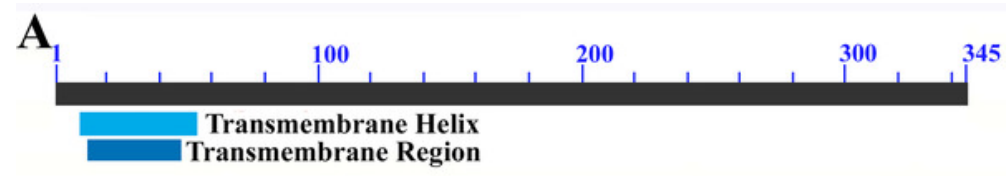

B

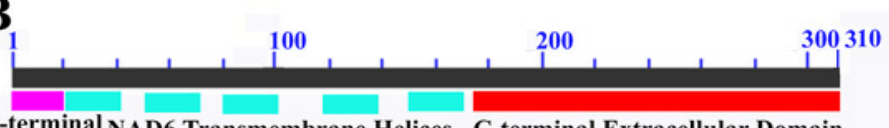

N-terminal NAD6 Transmembrane Helices C-terminal Extracellular Domain Intracellular

Domain

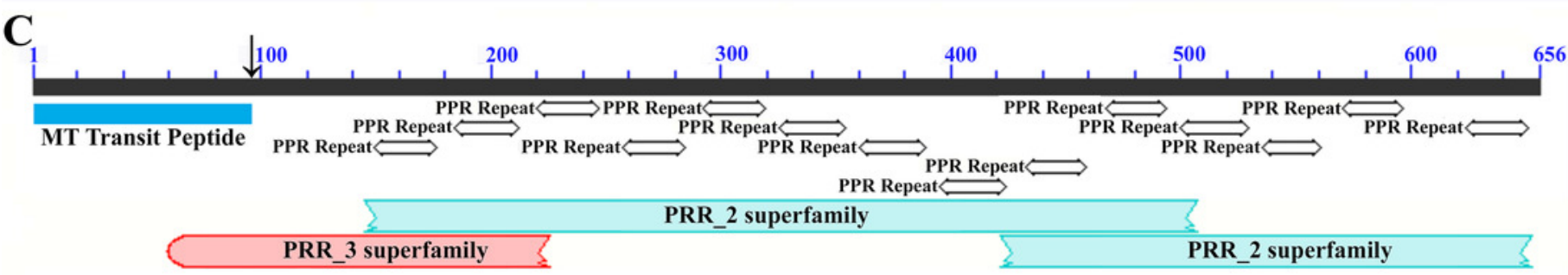

D

UBA_PL superfamily
UBQ superfamily
UBA_like
charged pocket

$\mathbf{E}$

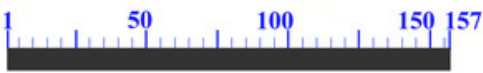

RRM_SF superfamily 


\section{Figure 7}

Mapping transcriptomic data to the sugarcane SP80-3280 mitochondrion and chloroplast.

Image showing the results of mapping transcriptomic reads to the sugarcane SP80-3280 mitochondrial and chloroplast genomes. A: SP80-3280 mitochondrial chromosome 1. B: SP80-3280 mitochondrial chromosome 2. C: SP80-3280 chloroplast genome. The y-axis represents $\log _{10}$ counts for transcript coverage at each base position within the genome. The $\mathrm{x}$-axis represents base position within the genome.
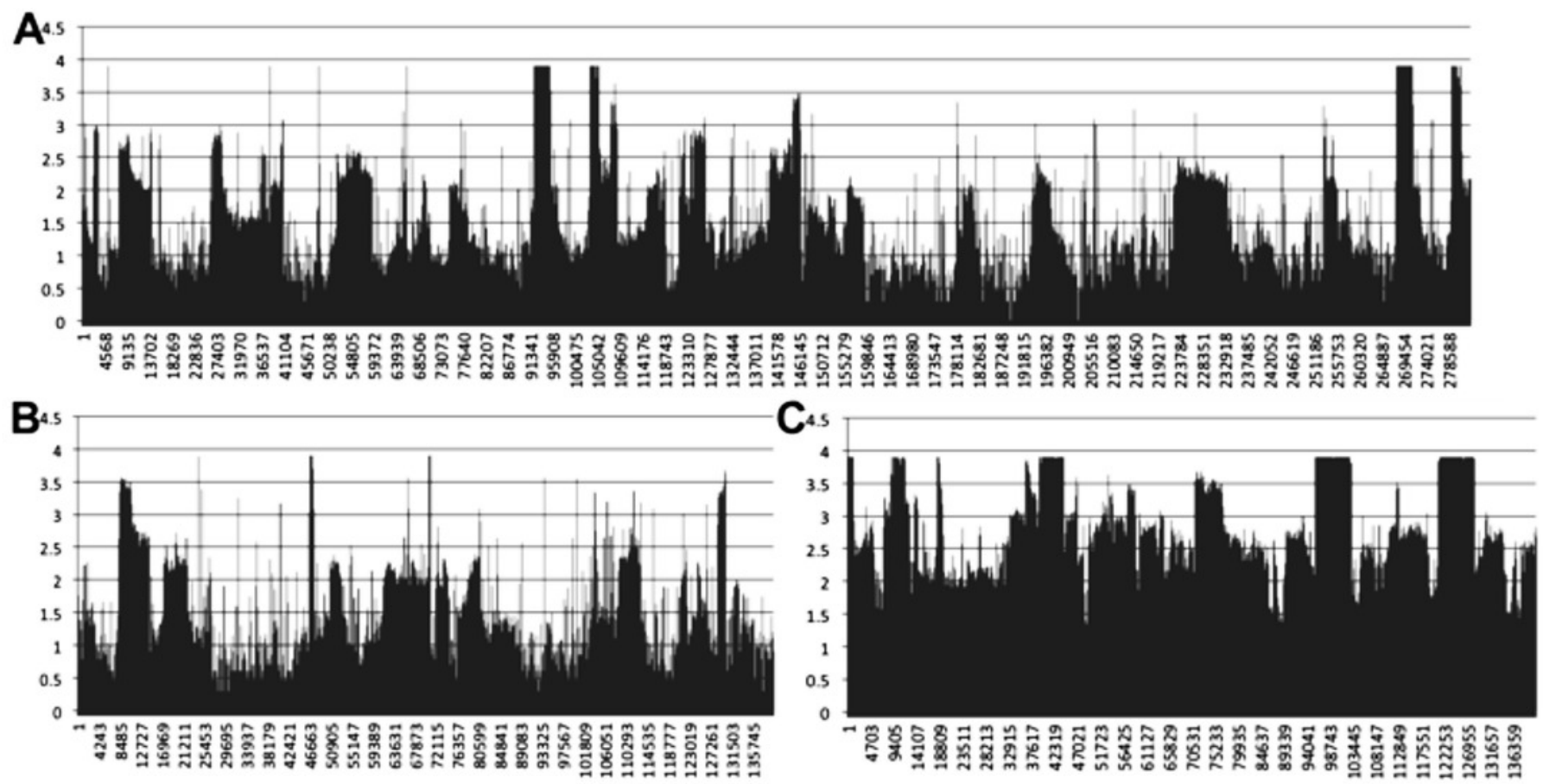


\section{Figure 8}

Structural comparisons of the sugarcane chloroplast and mitochondrial version of rbcL.

Superimposition and structural comparisons of the sugarcane chloroplast (mauve) and mitochondrial (green) version of the rbcL (rubisco large subunit). As can be seen, the structures are virtually identical and apart from truncations in the disordered amino $(\mathrm{N})$ and carboxyl (C) termini of the mitochondrial protein the only meaningful difference is the prediction of a helix centred on R86 in the chloroplast molecule and the prediction of a corresponding loop centred on Arg79 in the mitochondrial protein (shown with an arrow). However, as the sequences in the two regions are identical, this difference is almost certainly not meaningful. Otherwise, the structures are identical and active site amino acids are conserved, a strong indication that the sugarcane mitochondrial version of rbcL could be functional. 


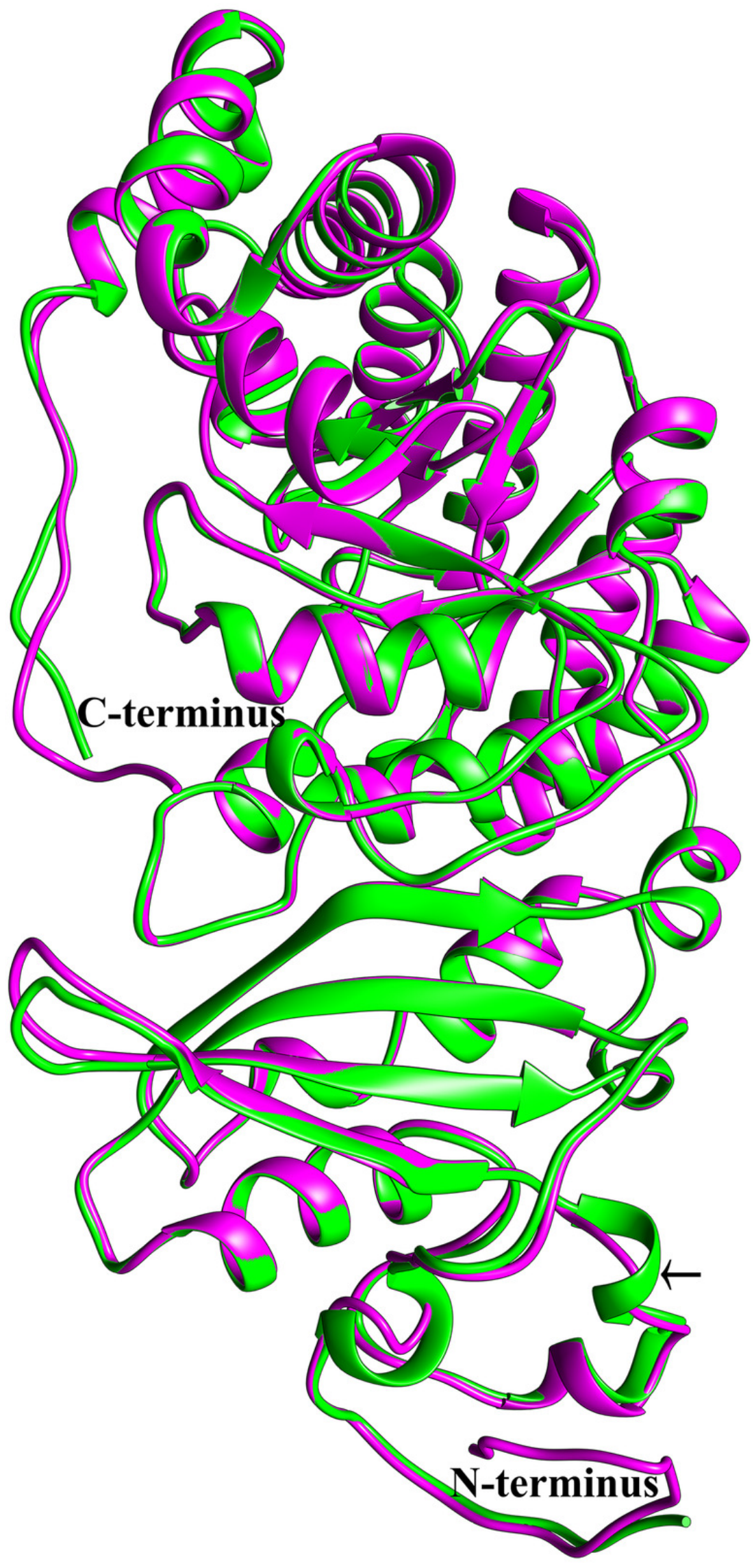




\section{Figure 9}

Origin of the sugarcane CMS2 cytoplasmic male sterility factor.

Image showing the origin of the sugarcane CMS2 cytoplasmic male sterility factor. CMS2 domains are mapped against a reference tree for the Andropogoneae (derived from Lloyd Evans, Joshi and Wang, 2019). Domains analysed and mapped are: red - nad6 5'; blue nad6 transmembrane region; yellow - cytoplasmic domain; brown - cytoplasmic domain 3' end. All regions are present in Chrysopogon zizanoides, but are gradually lost in the Tripsacinae. The full chimeric protein is present in all species from Coix lacryma-jobi to sugarcane. 


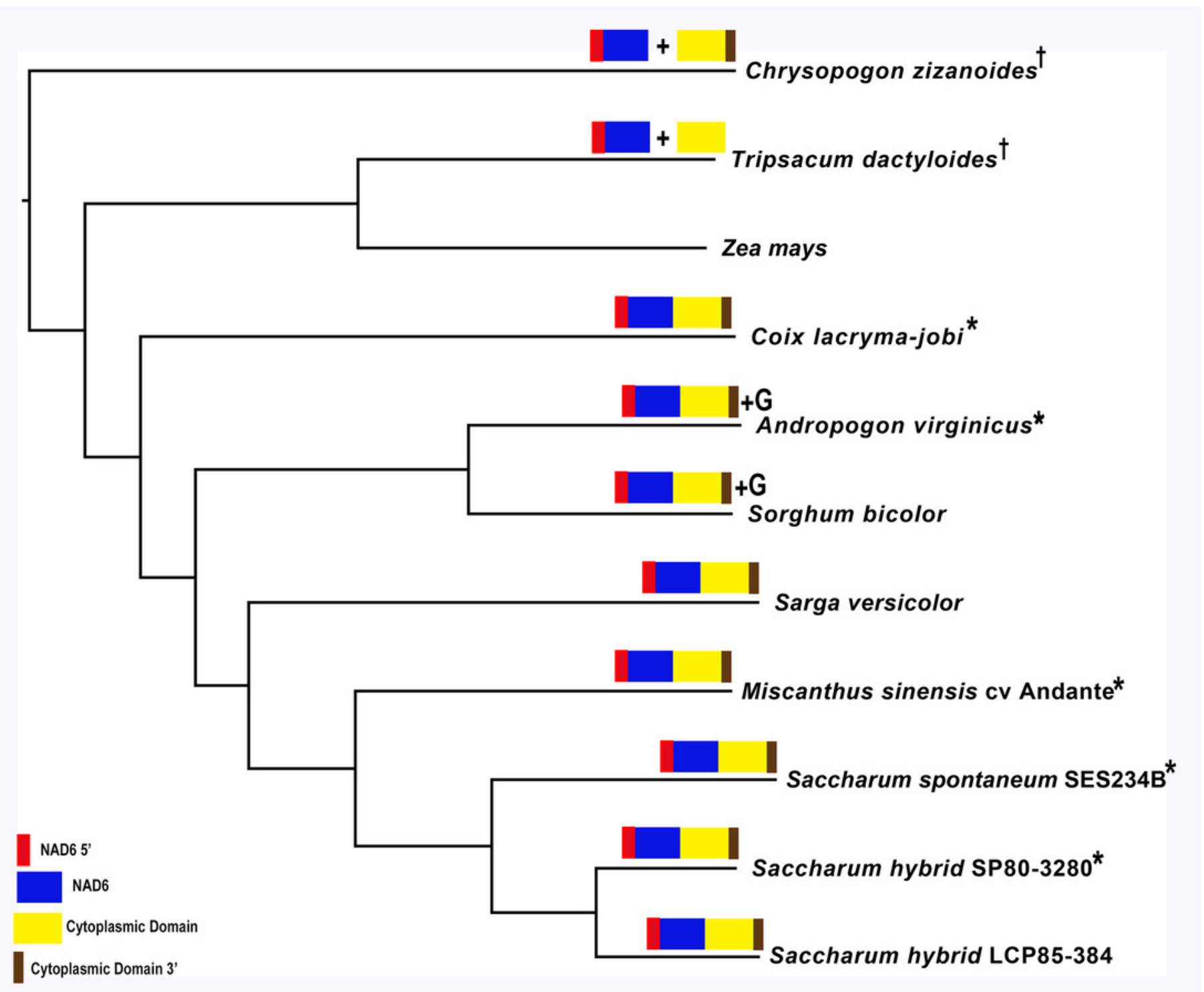




\section{Table $\mathbf{1}$ (on next page)}

Species, cultivar data with associated SRA accessions and publications.

A list of sugarcane cultivars, Saccharum species and other species analysed in this study along with the SRA files used for assembly and mapping and associated publications. 


\section{Saccharum hybrid Gnomic Data}

Saccharum hybrid SP80-3280

Saccharum hybrid LCP85-384

Saccharum hybrid RB72454

Saccharum hybrid LCP85-384

Saccharum hybrid SP70-1143

Saccharum hybrid R670 (PacBio)

Saccharum hybrid R670 (Illumina)

Saccharum hybrid transcriptomic data

Saccharum hybrid SP80-3280

Saccharum hybrid SP70-1143

Other Saccharum Species

Saccharum officinarum IJ76-514

Saccharum spontaneum SES234B

\section{Other Species Genomic Data}

Miscanthus sinensis cv Andante

Coix lacryma-jobi

Sarga versicolor

\section{Other Species Transcriptomic Data}

Chrysopogon zizanoides Sorghum bicolor BTx623 (random selection)

Sorghum bicolor BTx623 (polyA baited)

1

\section{SRA Accessions}

SRR1763296

SRR427145

SRR922219

SRR427145

SRR952331. SRR871521, SRR871522 and SRR871523

SRR8882845-SRR8882907

SRR7517604

PRJNA244522, SRR849062, SRR1974519,

SRR400035

SRR1104746, SRR1104748, SRR1104749, SRR619797

and SRR619800

SRR528718

SRR486146

SRR7121816

SRR427175

SRR2029676; SRR2167610; SRR2167619

SRR6002803; SRR2171885

ERR2097035; ERR2097063; ERR2097067;

ERR3063529 and ERR3087932

SRR5886574; SRR5921114; SRR5922762; SRR5925308; SRR5925309
Reference/Source

Riaño-Pachón \&

Mattiello, 2017

Grativol et al. 2014

Grativol et al. 2014

JGI Community Data

Grativol et al. 2014

JGI Community Data

JGI Community Data

Mattiello et al., 2015

Bottino et al., 2013;

Vargas et al., 2014

Berkman et al. 2014

JGI Community Data

Gifted by BeauSci Ltd Cambridge, UK BGI (WGS of 760 vascular plants)

JGI Community Data

Chakrabarty et al., 2015

Chungnam National University/BioCl

Cold Spring Harbor Laboratory

University of NebraskaLincoln 


\section{Table 2 (on next page)}

Capture primers and flanking sequence employed for mitochondrial isolation.

A list of sugarcane mitochondrial chromosome and bait regions with associated bait primers and 5' and 3' blocking flanking sequences used to capture different sugarcane mitochondrial conformations prior to sequencing. 
MT1

ccmC

$\mathrm{ccmFn}$

MT2

$\mathrm{ccmFc}$

cob

Putative master circl

p2a biotin-GACGCTTTGGTGACGAAGGTCACCGGGGTG

p3b biotin-GAAAGGAGACTGATCTTGACGTCGGCGTTG

4kbp Repeat

Probe

biotin-AGCATCCCACACCCGAAAGGTACCCCACAT

biotin-CCTTCCGCATTGGCGGCGAGTGGAGTGCCA

biotin-TACAAATCCATTTACGGATCTATATGCTCC

biotin-CAGCCAGATGAAGAAGACTGGCGCCTGCTA

p2a

biotin=ACCGGCAGCTAGCATCCCATCAGTAACCTA
5' block

TGCACCCAGGTAAATAAGGAACAAGATGAATACAGAAGTI

CGCATCCAGCAGAGCGAAGCAGCGTTCCATTCTTTTCGGC

TTTTTCCATTCGAGAAACGAGGAGCACGACTGAAGTGGCT

CAGAATGTACACCCAATGGATTATTTGATCCATATTGATG

ACTGATCCCCACTGGAGATTATATGAGGGGTCTTTGAAAC

AATCATTGGAATTTCCCATCTTTTGAAGCTCTGCTCCCAA

AgCACAGTATTTTTGTTCGTGCTCTGCACCACGTtTTTCC
3' block

TTCCCCGAAACCCCCCAGTCACTAACGTAAACAAAGTAGA CCATTCATCATTTTTGATCTACATAACCCAAAGCCCATAG

GAACTGGAAGTTCCAGAACTGGCGGCTGGTATACCACCAT AGGGGGAGTAAATGATGGAGACTAAAAAAACGATTTAAGG

TGGAAGGATTCCGTGGTAGTCTCTGACTCCCTCCAACTCA CCAACGAAAAACAAATTCGAACTTCAATGAAAAAACCAAA

GATATTTCtTGAGGGGGGCTGGCCATGGTTTTTTAACCCA 


\section{Table 3 (on next page)}

Primers used to amplify transcripts from the SP80-3280 and N22 sugarcane cDNA libraries.

A list of primers used to amplify potential restorer of function transcripts in both the SP80-3280 and N22 sugarcane cultivars. This table gives the gene names and types for the three potential CMS restorer of function transcripts identified in sugarcane. Also given are the forward and reverse primers used to amplify the transcripts, the length of the amplicons obtained and the melting temperatures $\left(T_{m}\right)$ for the primers. In addition, primers used to amplify the two mitochondrial genome stabilizing factors, RECG and RECA1 in SP80-3280 are detailed. 


\section{Gene}

ShRF1 PPR domain protein ShDSK2 ubiquitin domain

protein

ShGRP162 (glycine-rich

RNA-binding protein 3 )

Saccharum hybrid SP80-3280

RECG

Saccharum hybrid SP80-3280

RECA1

$\begin{array}{ll}\text { Left Primer } & \text { Right Primer } \\ \text { GCGCGACCGAGCTGCATTTCC } & \text { TCCCCTTtTGGCCATCTGCAGC } \\ \text { GGAACGAATCCGGACCGTC } & \text { TTGAAACCACCGGTTGGATTAG } \\ \text { GTGCGCGTAGCGCAGCGGGG } & \text { TGGCAGCACCAAGAAGCACCTT } \\ & \\ \text { CAGCCCAAACTTTTTTAGGTGGT } & \text { GGGTGAAGGACTGAAGGTGAAC } \\ \text { GGCATACGAGATCGGGACGGG } & \text { GCGCCTGATATTTTCCTTTGTTGG }\end{array}$

\section{Left Primer}

CATACGAGATCGGGACGGG

\section{Right Prime}

TATATTCCTTTGTTGG

$\begin{array}{rrl}\text { SP80 Amplicon Length } & \text { N22 Amplicon Length } & \text { Tm } \\ 2133 & 2136 & 72^{\circ} \mathrm{C} \\ 2313 & 2312 & 63^{\circ} \mathrm{C} \\ 1030 & 1030 & 72^{\circ} \mathrm{C} \\ & & \\ 3609 & & 62^{\circ} \mathrm{C} \\ 1758 & 68^{\circ} \mathrm{C}\end{array}$




\section{Table 4 (on next page)}

Comparisons of base-level differences in the mitochondria and chloroplasts of sugarcane cultivars to the SP80-3280 reference assemblies presented in this paper.

Analysis of base-by base comparisons of several sugarcane mitochondrial and chloroplast assemblies from different cultivars to the reference SP80-3280 assemblies presented in this paper. Mitochondrial data is given at the top and chloroplast data at the bottom. Columns represent: cultivar; total length of plastome; total number of substitutions; total number of insertions; total number of deletions. For mitochondria, positions of large direct and inverted repeats and the total number of small repeats are given. Numbers in brackets give substitutions corrected for transcript post-processing. The label ' $\mathrm{gb}$ ' means that the sequence is one downloaded from GenBank. 


\begin{tabular}{|c|c|c|c|c|c|c|c|}
\hline Mitochondria & Length & substitutions & insertions & deletions & $15 \mathrm{k}$ & Repeats & $\begin{array}{c}\text { small } \\
\text { repeats } \\
(<360 \\
\text { bp) }\end{array}$ \\
\hline SP80-3284 mt1 & 300960 & & & & $\begin{array}{l}9777- \\
285530\end{array}$ & $\begin{array}{l}45748- \\
174194 R\end{array}$ & 111 \\
\hline SP80-3284 mt2 & 144639 & & & & & & $19(55)$ \\
\hline IJ76-514 mt1 & 300995 & 470 & 25 & 8 & $\begin{array}{l}98560- \\
289970\end{array}$ & $\begin{array}{l}45945- \\
174355 R\end{array}$ & 129 \\
\hline IJ76-514 mt2 & 144926 & 261 & 32 & 8 & 97558- & 45748- & $120(56)$ \\
\hline RB72454 mt1 & 300828 & 79 & 3 & 7 & 285312 & 174074R & 134 \\
\hline RB72454 mt2 & 144692 & 67 & 8 & 1 & 97691- & 46049- & $52(55)$ \\
\hline LCP85-384 mt1 & 300775 & 126 & 1 & 3 & 285426 & 173891R & 142 \\
\hline LCP85-384 mt2 & 144679 & 105 & 7 & 0 & 98097- & 50210- & $47(54)$ \\
\hline R570 mt1 & 300786 & 59 & 0 & 8 & 285846 & 174517R & 107 \\
\hline $\mathrm{R} 570 \mathrm{mt} 2$ & 144736 & 29 & 10 & 1 & & & $54(40)$ \\
\hline $\begin{array}{l}\text { Khon Kaen } 3 \text { mt1 } \\
\text { (gb) } \\
\text { Khon Kaen } 3 \text { mt2 }\end{array}$ & 300784 & 40 & 5 & 11 & $\begin{array}{l}97558- \\
288181\end{array}$ & $\begin{array}{l}45748- \\
174045 R\end{array}$ & 107 \\
\hline (gb) & 144648 & 12 & 1 & 0 & 97674- & 45748- & $19(55)$ \\
\hline SP70-1143 mt1 & 300972 & $118(63)$ & 5 & 10 & 285433 & 174192R & 142 \\
\hline SP70-1143 mt2 & 144676 & $44(27)$ & 8 & 0 & & & $47(55)$ \\
\hline $\begin{array}{l}\text { Chloroplasts } \\
\text { SP80-3280 } \\
\text { (Genomic) }\end{array}$ & 141181 & & & & & & \\
\hline $\begin{array}{l}\mathrm{SP} 80-3280 \mathrm{cp}(\mathrm{gb}) \\
\mathrm{SP} 80-3280 \\
\text { transcriptomic }\end{array}$ & $\begin{array}{l}141182 \\
141181\end{array}$ & $\begin{array}{r}8 \\
45(0)\end{array}$ & 0 & 1 & & & \\
\hline IJ76-514 & 141176 & 26 & 2 & 5 & & & \\
\hline NCo310 & 141182 & 5 & 0 & 0 & & & \\
\hline RB72454 & 141181 & 7 & 0 & 0 & & & \\
\hline R570 & & 7 & 0 & 0 & & & \\
\hline Q155 & 141181 & 0 & 0 & 0 & & & \\
\hline Q165 & 114181 & 2 & 0 & 0 & & & \\
\hline RB867515 & 141181 & 0 & 0 & 0 & & & \\
\hline $\begin{array}{l}\text { SP70-1143 } \\
\text { SP70-1143 } \\
\text { (transcriptomic) }\end{array}$ & $\begin{array}{l}141181 \\
141181\end{array}$ & $\begin{array}{r}2 \\
43(2)\end{array}$ & 0 & $\begin{array}{l}0 \\
0\end{array}$ & & & \\
\hline LCP85-384 & 141185 & 2 & 1 & 0 & & & \\
\hline
\end{tabular}

1 


\section{Table 5 (on next page)}

Expression analysis of Mitochonrial rbcL in the sugarcane cultivar SP80-3280.

Analysis of transcript expression for the mitochondrial copy of $r b c L$ in the sugarcane cultivar SP80-3280 compared with nad6, with a random 1000bp non-coding mitochondrial region used as a reference. Transcript matches were normalized against gene length and expressed as fold change in comparison to the non-coding regions. 


\section{Gene/Region}

nad6

non-coding region

rbcL C-termnus

Fold Difference nad6

1

Fold Difference rbcL
Number of Mapped Gene Length Reads

804

1000

231

181.79

15.12
Normalized Read

Counts

29.81

0.164

2.48 